\title{
Thermal Conduction in Higher-Dimensional Sphere and Application to Fluid Rotation
}

Osuga $\mathrm{T}^{*}$

Center for Frontier Medical Engineering, Chiba University, Japan

*Corresponding author: Toshiaki Osuga, Center for Frontier Medical Engineering, Chiba University, Yayoi, Inage, Chiba 263-8522, Japan, Tel: 043-290-3123; Fax 043-

290-3123; Email: artisankoshik@yahoo.co.jp

\section{Research Article}

Volume 2 Issue 3

Received Date: July 19, 2017

Published Date: July 25, 2017

DOI: $10.23880 /$ nnoa- 16000125

\section{Abstract}

When two parallel disks, a cylinder, and a sphere having a static fluid within start to rotate, the increase in the angular velocity of the laminar fluid layers starting coaxial rotation was found to be mathematically equivalent to the thermal conduction of one-, four-, and five-dimensional spheres heated from their surfaces, respectively. The thermal conduction of an $n$-dimensional sphere for $4 \leq n \leq 100$ was numerically simulated using a time step $\Delta t$ and radial division $\Delta r$. The growth period required to achieve a final flat temperature profile was defined as the time when the center temperature reached 99/100 of the surface temperature. The growth period was numerically determined to be proportional to $n^{-1.25}$, which was analytically supported by the thermal conduction of $n$-dimensional cubes inscribed within and circumscribing an $n$-dimensional sphere. Because the exponential time change in the surface temperature gradient was almost similar for the spherical heating of 1-8-dimensional spheres, the entire thermal transport in the $n$-dimensional sphere for $1 \leq n \leq 8$ was numerically determined to be similar with respect to the growth period, which was supported by the analytical solutions for spheres with $1 \leq n \leq 3$ by regarding slab and cylindrical thermal conduction as the heating modes of one- and two-dimensional spheres, respectively. The observed attenuation periods of fluid rotation in cylinder and spherical flasks were supported by the numerically determined growth periods of the thermal conduction of four- and five-dimensional spheres, where the rotation in the cylinder should be treated as disk rotation when the height is less than the radius.

Keywords: Von Neumann Stability Analysis; Lax-Rightmyer Theorem; Courant-Friedrichs-Lewy Condition; Courant Number; Explicit Method; Forward Difference; Fourier Number; Staggered Grid; Hypercube

\section{Introduction}

An infinite straight cylinder submerged in a fluid is assumed to suddenly start to move in the axial direction with a constant velocity $V$ at $t=0$, where $t$ is the time. When no vortexes are generated, the translational laminar flow layers (flows in the axial direction with cylindrical symmetry) are represented by a telescope 


\section{Nanomedicine \& Nanotechnology Open Access}

model [1], grow from the inner surface of the cylinder towards its central axis, and finally approach a flat flow profile [2]. The growth period $t_{\mathrm{g}}$ is defined as the time when the difference between the fluid velocity along the cylinder axis and $V$ becomes less than $(1 / 100) V$ and expressed as $t_{\mathrm{g}}=5 R^{2} \rho / 2.4^{2} \mu$, where $R, \rho$, and $\mu$ are the inner radius of the cylinder and the density and viscosity of the fluid, respectively. When the cylinder and fluid move in the axial direction at a similar velocity $V$ for $t>t_{\mathrm{g}}$, the cylinder suddenly stops its motion. The attenuation of the flow profile inside the cylinder toward the completely terminated flow is similar to the growth of the flow profile. Thus, the attenuation period $t_{\mathrm{a}}$ is defined and expressed in a similar manner as $t_{\mathrm{g}}$. $t_{\mathrm{g}}$ and $t_{\mathrm{a}}$ are helpful for evaluating the time resolution of the body motion constructed by the semicircular canal using a lymph fluid rotation flow. Assuming that $\rho / \mu$ of the lymph fluid is $1.25 \times 10^{6}$ at $37^{\circ} \mathrm{C}$ and $R$ of the semicircular canal is $0.2 \mathrm{~mm}$ [3], $t_{\mathrm{g}}$ is $43.4 \mathrm{~ms}$. When our body rotates within $43.4 \mathrm{~ms}$, it is difficult for the semicircular canal to detect the body rotation. A cylindrical water tank with a diameter of 20 $\mathrm{cm}$ is used to detect a non-uniformity in the magnetic field for proton magnetic resonance imaging (MRI) [4]. Owing to the acceleration during the placement of the water tank in the MRI apparatus, the water begins to rotate. The static field measurement should be postponed until the water has stopped rotating. The steady torque solutions of a laminar flow surrounded by two concentric cylindrical and spherical shells rotating coaxially were applied to the fluid viscosity measurement $[5,6]$ and analysis of Brownian rotational motion [7-9], respectively. The viscosity of a fluid is measured by the torque generated in the inner cylinder when the outer cylinder rotates at a constant angular velocity. The measurement of the viscosity should start after the torque reaches a steady state. Therefore, the evaluation of $t_{\mathrm{g}}$ and $t_{\mathrm{a}}$, which are the transient times moving towards the steady state, is often required in scientific measurements [1].

The analytical solutions of the growth of the temperature profile approaching a steady temperature profile in a planar slab, cylinder, and sphere from their surfaces have been obtained using Fourier series and exponentially decreasing terms [10-12]. The thermal conduction of the slab, cylinder, and sphere is regarded as the heating modes of one-, two- and three-dimensional spheres, respectively. The coaxial laminar fluid flows generated by a rotating cylinder and sphere are found to be mathematically equivalent to the thermal conduction of four- and five-dimensional spheres heated from the surface in this study. Because no analytical solutions of the inward thermal conduction of $n$-dimensional spheres for $n \geq 4$ have been obtained, numerical analyses for an $n$ dimensional sphere up to $n=100$ were performed, and the growth periods required to achieve a steady flat temperature profile in the sphere were found to be expressed by a concise power law in terms of the number of dimensions $n$ [12]. Although the thermal conduction equation for a planar geometry has only a diffusion term, that for a spherical geometry has both diffusion and advection terms [10-12].

In numerical simulations, the time and space should be divided into minute increments of $\Delta t$ and $\Delta r$, respectively. The determination of $\Delta t$ is restricted by $\Delta r$ in a different manner in the diffusion and advection equations. The use of $\Delta t$ and $\Delta r$ outside any restriction will lead to unstable and meaningless simulations. The condition relating $\Delta t$ and $\Delta r$ in the diffusion equation is the von NeumannRightmyer (NR) condition [13-14], and that in the advection equation is the Courant-Friedrichs-Lewy (CFL) condition [15-16]. The properties of diffusion and advection are stronger for lower and higher $n$, respectively, for $n$-dimensional spherical thermal conduction. Thus, the numerical difference scheme is strongly affected by the NR and CFL conditions for lower and higher $n$, respectively.

Currently, there are no physical meanings for $n$ dimensional spherical heating for $n>5$, whereas there is a physical meaning for fluid rotation for four- and fivedimensional spherical heating. However, the numerical simulation of $n$-dimensional spherical heating for up to $n=100$ should be conducted because any $n$-dimensional spherical heating for $n>1$ includes a diffusion and advection equation, and the determination of a systematic law for $n$ assures the numerical accuracy of four- and fivedimensional spherical heating. We reveal that the nonsteady force couples of a cylinder or sphere rotating in a fluid, which were derived from numerical simulations of four- and five-dimensional spherical heating, are required for the analysis of the rotational Brownian motion [9]. The purpose of this study is to investigate the numerical accuracy of $n$-dimensional spherical thermal conduction in order to increase the exactness of four- and fivedimensional spherical heating applied to fluid rotation and Brownian motion.

\section{Spherical Thermal Conduction Characterized by Advection and Diffusion}




\section{Nanomedicine \& Nanotechnology Open Access}

Heat Transfer between Adjacent $n$-Dimensional Spherical Shells: The inward thermal conduction of a sphere heated from the surface shown in Figure 1a is considered. The entire volume ${ }^{n V}$ and surface area ${ }^{n} S$ of the $n$-dimensional sphere with a radius of $a$ and the relation between ${ }^{n} V$ and ${ }^{n} S$ are given by [17].

$$
{ }^{n} V=\frac{a^{n} \pi^{\frac{n}{2}}}{\Gamma\left(\frac{n}{2}+1\right)},{ }^{n} S=\frac{n a^{n-1} \pi^{\frac{n}{2}}}{\Gamma\left(\frac{n}{2}+1\right)},{ }^{n} V=\int_{0}^{a}{ }^{n} S d a,
$$

Where the function $\rho$ satisfies $\Gamma(n+1)=n \Gamma(n)$, and $\Gamma(1 / 2)=\pi^{1 / 2}$. The $n$-dimensional sphere is divided into a series of thin concentric shells with spherical symmetry and a thickness of $\Delta r$ as shown in Figure 2. Thermal conduction is calculated from the heat transfer between adjacent shells. The shell boundaries $r_{k}$ are subscripted with 0 to max such that $r_{0}=0.0$ and $r_{\max }=a$. The symbols $r_{k-1}, r_{k-1 / 2}$, and $r_{k}$ denote the inner radius $(k-1) \Delta r$, the central radius $(k-1 / 2) \Delta r$, and the outer radius $k \Delta r$ of the $k$-th shell, respectively. The number $k$ assigned to each shell coincides with that assigned to its outer boundary so that these positions are numbered from 1 to max. The temperatures of the shells are denoted $T_{1}$ to $T_{\max }$. The temperature of each shell is defined at the center of the shell, i.e., at $r=r_{k-1 / 2}$. Before $t=0$, the temperatures of all shells $T_{i}(i=1 \rightarrow \max )$ are set to zero as the initial condition. After $t=0$, the temperature in the surface shell is maintained at a fixed temperature $T_{\mathrm{s}}$, i.e., $T_{\max +1}=T_{\mathrm{s}}$, and the inward thermal conduction transfers heat from the surface toward the center. The inward conduction requires central symmetry: $T_{0}=T_{1}$. The volume ${ }^{n} V_{k}$, inner surface area ${ }^{n} S_{k-1}$, and outer surface area ${ }^{n} S_{k}$ of the $k$-th spherical shell in the $n$-dimensional sphere are derived from Equation (2.1) as

$$
{ }^{n} V_{k}=\frac{\pi^{\frac{n}{2}}\left(r_{k}^{n}-r_{k-1}^{n}\right)}{\Gamma\left(\frac{n}{2}+1\right)},{ }^{n} S_{k}=\frac{n \pi^{\frac{n}{2}} r_{k}^{n-1}}{\Gamma\left(\frac{n}{2}+1\right)},{ }^{n} S_{k-1}=\frac{n \pi^{\frac{n}{2}} r_{k-1}^{n-1}}{\Gamma\left(\frac{n}{2}+1\right)}
$$

In the $k$-th spherical shell $\left(k=1 \rightarrow k_{\max }\right)$, the volumetric heating is derived from the thermal conduction between adjacent shells. The progression in $t$ is denoted by the positive integer $m$. The present and next times are expressed using $m$ as $m \Delta t$ and $(m+1) \Delta t$, respectively, where $\Delta t$ is the minute time step. The heat influx $\Gamma_{\mathrm{h}}$ is generated from the adjacent $(k+1)$-th shell to the $k$-th shell, which is proportional to the temperature gradient and surface area, expressed as $\Gamma_{\mathrm{h}}=\lambda^{n} S_{k}\left(T_{k+1}{ }^{m}-T_{k^{m}}\right) / \Delta r$, where $\lambda$ is the thermal conductivity, measured in watts per meter per degree Kelvin. The temperature $T_{k}{ }^{m+1}$ of the $k$-th shell at the next time step $(m+1) \Delta t$ is determined by three terms at the present time step $m \Delta t$ : the temperature of the $k$-th shell $T_{k}^{m}$, the heat transfer from the $(k+1)$-th shell ${ }^{n} S_{k}\left(T_{k+1}{ }^{m}-T_{k}{ }^{m}\right) / \Delta r$, and the heat transfer from the $(k-1)$-th shell ${ }^{n} S_{k-1}\left(T_{k-1}{ }^{m}-T_{k}{ }^{m}\right) / \Delta r$, which are expressed with a difference scheme with the aid of Equation (2.2) as

$\rho C_{p}\left(r_{k}^{n}-r_{k-1}^{n}\right) \frac{T_{k}^{m+1}-T_{k}^{m}}{\Delta \mathrm{t}}=\lambda n r_{k}^{n-1} \frac{T_{k+1}^{m}-T_{k}^{m}}{\Delta \mathrm{r}}+\lambda n r_{k-1}^{n-1} \frac{T_{k-1}^{m}-T_{k}^{m}}{\Delta \mathrm{r}}$

Where $\rho C_{p}$ is the volumetric specific heat measured in joules per cubic meter per degree Kelvin. Equation (2.3) is arranged as a difference scheme as

$$
\frac{T_{k}^{m+1}-T_{k}^{m}}{\Delta}=\chi \frac{n}{r_{k}^{n}-r_{k-1}^{n}}\left(r_{k}^{n-1} \frac{T_{k+1}^{m}-T_{k}^{m}}{\Delta \mathrm{r}}-r_{k-1}^{n-1} \frac{T_{k}^{m}-T_{k-1}^{m}}{\Delta \mathrm{r}}\right)
$$

Where $\chi$ is the thermal diffusivity $\lambda /\left(C_{p} \rho\right)$ measured in meters squared per second. Equation (2.4) is adequate for the numerical calculation of the thermal conduction of an $n$-dimensional sphere. The volume of the $k$-th spherical shell is proportional to $\left(r_{k}^{n}-r_{k-1}{ }^{n}\right)$, which is approximated as $n r_{k-1 / 2^{n-1}} \Delta r$. Thus, Equation (2.4) converges to the following partial differential equation for infinitely small $\Delta r$ and $\Delta t$ :

$$
\frac{\partial T}{\partial t}=\chi \frac{1}{r^{n-1}} \frac{\partial}{\partial r} r^{n-1} \frac{\partial T}{\partial r}
$$

The form of Equation (2.5) is appropriate for numerical calculation using the difference scheme in Equation (2.4). Equation (2.5) can be equivalently converted into the following equation [10-12]:

$$
\frac{\partial T}{\partial t}=\chi\left(\frac{\partial^{2} T}{\partial r^{2}}+\frac{n-1}{r} \cdot \frac{\partial T}{\partial r}\right)
$$

Equation (2.6) can compare the effects of the diffusion term $\partial^{2} T / \partial r^{2}$ and the advection term $\partial T / \partial r$ appearing in the thermal conduction equation for $n \geq 2$, where the effect of the advection term becomes greater as $n$ increases, which is defined as a spherical geometry effect. Although thermal conduction mainly progresses 


\section{Nanomedicine \& Nanotechnology Open Access}

by the planar heat transfer term $\partial^{2} T / \partial r^{2}, \partial T / \partial r$ is related to spherical conversion and transfers heat in a different manner, as described in Secs. 3 and 4.

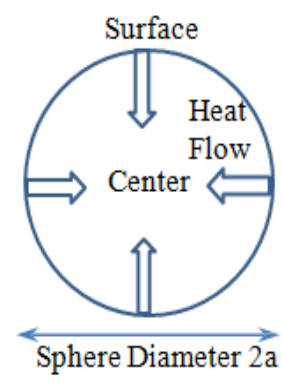

(a)

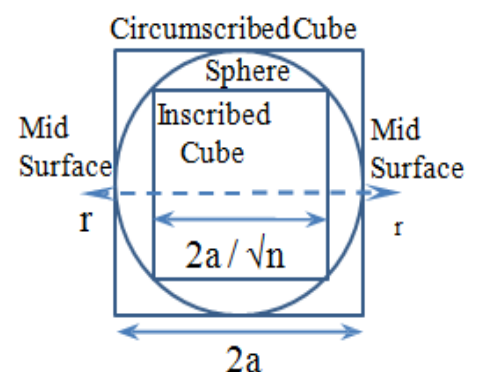

(b)
Figure 1: (a) Inward thermal conduction of an $n$ dimensional sphere heated from the hot surface toward the cold center. (b) An $n$-dimensional cube with a radius of a circumscribing an $n$-dimensional sphere with a radius of $a$ and an $n$-dimensional cube with a radius of $a n^{-1 / 2}$ inscribed within an $n$ dimensional sphere.

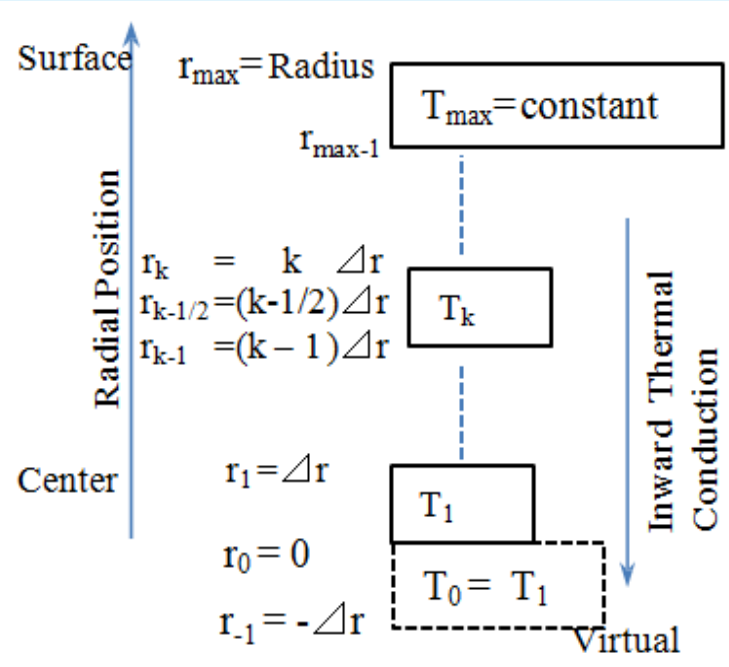

Figure 2: Numbering of the spherical shells and their boundaries.

\section{Convex Upward Temperature Profile Formed by the Advection Term}

Planar heating from a single plane source located at $r=0$, as shown in Figure 3a, is considered. The boundary conditions are expressed as follows:

$$
T(r, t<0)=T_{c} \quad \text { and } \quad T(r=a, \quad t \geq 0)=T_{s} .
$$

Before $t=0$, both the surrounding volume and plane have a uniform temperature $T(r, t)=T_{\mathrm{c}}$. After $t=0$, the temperature at the plane is maintained at a constant value of $T_{\mathrm{s}}\left(>T_{\mathrm{c}}\right)$. Consequently, the surrounding volume $(r>0)$ is heated from the plane for $t \geq 0$. Hereafter, it is assumed that $T_{\mathrm{c}}=0$ and $T_{\mathrm{s}}=1$. The planar thermal conduction equation is derived from Equation (2.6) with for $n=1$ as

$$
\frac{\partial T}{\partial t}=\chi \frac{\partial^{2} T}{\partial r^{2}}
$$

By applying the boundary conditions in Equation (2.7) to Equation (2.8), the growth of the temperature profile is analytically derived as $[1,18,19]$.

$$
\begin{aligned}
& T(r, t)=T_{s}[1-\operatorname{erf}(\eta)], \quad \eta=\frac{r}{2 \sqrt{\chi t}} \\
& \operatorname{erf}(\eta)=\frac{2}{\sqrt{\pi}} \int_{0}^{\eta} e^{-p^{2}} d p\left[\approx \frac{2}{\sqrt{\pi}}\left(\eta-\frac{\eta^{3}}{3}+\frac{\eta^{5}}{10}-\frac{\eta^{7}}{42}+\frac{\eta^{9}}{216}+\cdots\right)\right]
\end{aligned}
$$

The spatial differential on the right-hand side of Equation (2.8) contains only the diffusion term $\partial^{2} T / \partial r^{2}$. The temperature at any place except the boundary should increase with time, i.e., $\partial T / \partial t>0$, in the case of planar heating. Therefore, planar heating always yields a convex downward temperature profile with no inflection points, i.e., $\partial^{2} T / \partial r^{2}>0$ for $r>0$.

Although the diffusion term $\partial^{2} T / \partial r^{2}$ appears for both the planar and spherical geometries for $n \geq 1$, the advection term $\partial T / \partial r$ appears only for the spherical geometry for $n \geq 2$, as shown in Equation (2.6). The elimination of the diffusion term $\partial^{2} T / \partial r^{2}$ from Equation (2.6) yields the following hyperbolic equation:

$$
\frac{\partial T}{\partial t}-\chi \frac{n-1}{r} \cdot \frac{\partial T}{\partial r}=0, \text { for } n \geq 1
$$




\section{Nanomedicine \& Nanotechnology Open Access}

The hyperbolic equation $\partial T / \partial t+u \partial T / \partial r=0$ transfers heat with the heat transport velocity $u$. Thus, the temperature profile shifts in position with $u$. Equation (2.10) transfers heat from the planar heat source with the negative (centripetal) velocity $u=-\chi(n-1) / r$ for $n \geq 2$. Thus, the step-like temperature profile formed at the surface just after $t=0$ shifts in position toward the center with the centripetal velocity and forms a convex upward temperature profile. The temperature profile around the center is always convex downward. Therefore, the temperature profile for inward thermal conduction for $n \geq 2$ is composed of upward and downward convex curves and an inflection point.

\section{Fluid Torque Equations Equivalent to One-, Four- and Five-Dimensional Heating}

Fluid Rotation in a Cylinder Described as FourDimensional Spherical Heating: When the cylinder starts to rotate about the $z$ axis with a constant angular velocity $\Omega$ at $t=0$, the cylindrical laminar flow layers rotate coaxially with the angular velocity $\omega(r)$ and diffuse from the surface cylindrical shell to the central axis. Finally, their velocity approaches $\Omega$. Each laminar flow layer is equivalent to the $k$-th cylindrical shell $\left(k=1 \rightarrow k_{\max }\right)$ with the angular velocity $\omega_{k}$ and flow velocity $v_{k}=r_{k-1 / 2} \omega_{k}$. The flow grows owing to the torque acting between adjacent shells. The torque $\sigma$ acting on the $k$-th shell from the adjacent $(k+1)$-th shell is expressed as the product of the radius $r_{k}$ as the length of the moment, the contacting surface area $2 \pi r_{k} \Delta z$, and the velocity shear drag $\mu\left(r_{k} \omega_{k+1} m-r_{k} \omega_{k^{m}}\right) / \Delta \rho$ and expressed as $\sigma=\mu r_{k} 2 \pi r_{k} \Delta z\left(r_{k} \omega_{k+1}{ }^{m}-r_{k} \omega_{k}{ }^{m}\right) / \Delta \mathrm{r}$, where $\Delta z$ is the length of the cylindrical shell. It should be noted that the flow velocity shear generated at the boundary radius $r=r_{k}$ originates from $r_{k} \omega_{k+1}{ }^{m}-r_{k} \omega_{k}^{m}$; the velocity difference crossing the boundary because $\omega$ and the velocity shear are defined at the center of the shell at $r=r_{k+1 / 2}$ and the boundary between the shells at $r=r_{k}$, respectively. The total torque $N=N_{k}$ upper $+N_{k}$ lower acting on the $k$-th shell is determined by the upper torque $N_{k}^{\text {upper }}$ from the $(k+1)$-th shell and the lower torque $N_{k}^{\text {lower }}$ from the $(k-1)$-th shell calculated at the present time step $m \Delta t$, where $N_{k}^{\text {upper }}=\mu r_{k} \quad 2 \pi r_{k}\left(r_{k} \omega_{k+1}^{m}-r_{k} \omega_{k}^{m}\right) / \Delta r$, and $\quad N_{k}^{\text {lower }}=\mu r_{k}$. ${ }_{1} 2 \pi r_{k-1}\left(r_{k-1} \omega_{k-1}^{m}-r_{k-1} \omega_{k}^{m}\right) / \Delta r$. Because the moment of inertia $I_{k}$ of the $k$-th shell rotating along the $z$ axis is $I_{k}=\rho \pi\left(r_{k}^{2}-r_{k-1}^{2}\right) \cdot r_{k-\frac{1}{2}}^{2} \Delta z, \omega_{k}$ of the $k$-th shell is determined by a torque equation as

$$
I_{k} \frac{\partial \omega_{k}}{\partial \mathrm{t}}=N_{k}^{\text {upper }}+N_{k}^{\text {lower }}
$$

Equation (2.11) is transformed into the difference scheme as follows, where $\omega_{k}{ }^{m+1}$ at the next time step $(m+1) \Delta t$ is determined by the quantities at the present time step $m \Delta t$ :

$$
\begin{aligned}
& \rho \pi\left(r_{k}^{2}-r_{k-1}^{2}\right) \cdot r_{k-\frac{1}{2}}^{2} \Delta \mathrm{z} \cdot \frac{\omega_{k}^{m+1}-\omega_{k}^{m}}{\Delta \mathrm{t}} r_{k-\frac{1}{2}}^{2} \\
& =\mu 2 \pi r_{k} \Delta \mathrm{z} \cdot \frac{\left(r_{k} \omega_{k+1}^{m}-r_{k} \omega_{k}^{m}\right)}{\Delta \mathrm{r}} r_{k}+\mu 2 \pi r_{k-1} \Delta \mathrm{z} \cdot \frac{\left(r_{k-1} \omega_{k-1}^{m}-r_{k-1} \omega_{k}^{m}\right)}{\Delta \mathrm{r}} r_{k-1}
\end{aligned}
$$

Using $r_{k}{ }^{j}-r_{k-1}{ }^{j} \approx j r_{k-1 / 2^{j-1}} \Delta r$, Equation (2.12) is transformed into

$\rho\left(\begin{array}{c}4 \\ r_{k}-r_{k-1}^{4}\end{array}\right) \frac{\omega_{k}^{m+1}-\omega_{k}^{m}}{\Delta \mathrm{t}}=\mu 4 r_{k}^{3} \frac{\left(\begin{array}{c}m \\ \omega_{k+1}-\omega_{k}^{m}\end{array}\right)}{\Delta \mathrm{r}}+\mu 4 r_{k-1}^{3} \frac{\left(\begin{array}{c}m \\ \omega_{k-1}-\omega_{k}^{m}\end{array}\right)}{\Delta \mathrm{r}}$

Equation (2.13) is equivalent to the thermal conduction of a four-dimensional sphere in Equation (2.3). The growth of the angular velocity profile is analogous to spherical heating from a surface for $n=4$. The exchange of $T(r)$ with $\omega(r)$ and $\chi$ with $v(=\mu / \rho)$ in Equation (2.6) for $n=4$ yields the growth shown in Figure 4(a4). The fluid between two coaxial cylinders having radii of a and $b$ starts to rotate following the coaxial rotations of the inner and outer cylinders, where $\omega$ is $\Omega$ and $\omega_{\text {out }}$ at $r=a$ and $b$, respectively. After a growth period, the stationary angular velocity profile is derived from Equation (2.6) for $n=4$ by setting $\partial / \partial t=0$ as [6]

$$
\omega=\frac{b^{2}}{\left(b^{2}-a^{2}\right)}\left[\left(\Omega-\omega_{\text {out }}\right)\left(\frac{a}{r}\right)^{2}-\Omega\left(\frac{a}{b}\right)^{2}+\omega_{\text {out }}\right]
$$

When the cylinder with a radius of $a$ rotates at $\omega=\Omega$ immersed in an infinitely wide static fluid, the angular velocity profile for $r \geq a$ is given as $\omega=(a / r)^{2} \Omega$ by letting $b / a \rightarrow \infty$ and setting $\omega_{\text {out }}=0$ in Equation (2.14). The force couple for the cylinder at a unit length for maintaining $\Omega$ is given as $4 \pi a^{2} \mu \Omega$, which is derived from $2 \pi a \times a \times\left(\partial(r \omega) /\left.\partial r\right|_{r=a}\right)$. 


\section{Nanomedicine \& Nanotechnology Open Access}

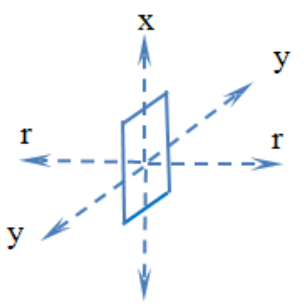

(a) Plane

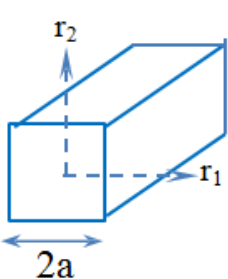

(c) Duct

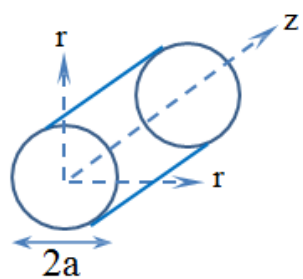

(e) Cylinder

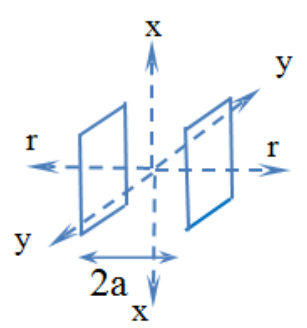

(b) Slab

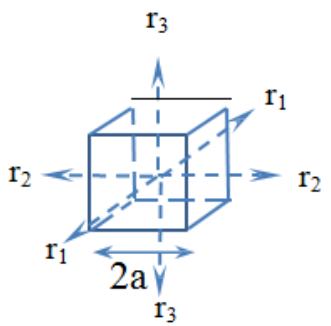

(d) Cube

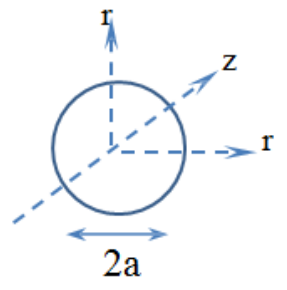

(f) Sphere
Figure 3: Coordinate systems of a (a) plane, (b) slab (one-dimensional sphere), (c) rectangular duct (twodimensional cube), (d) cube, (three-dimensional cube), (e) cylinder (two-dimensional sphere), and (f) sphere (three-dimensional sphere). The diameters of the spheres and cubes are $2 \mathrm{a}$.
Osuga T. Thermal Conduction in Higher-Dimensional Sphere and Application to Fluid Rotation. Nanomed Nanotechnol 2017, 2(3): 000125 .

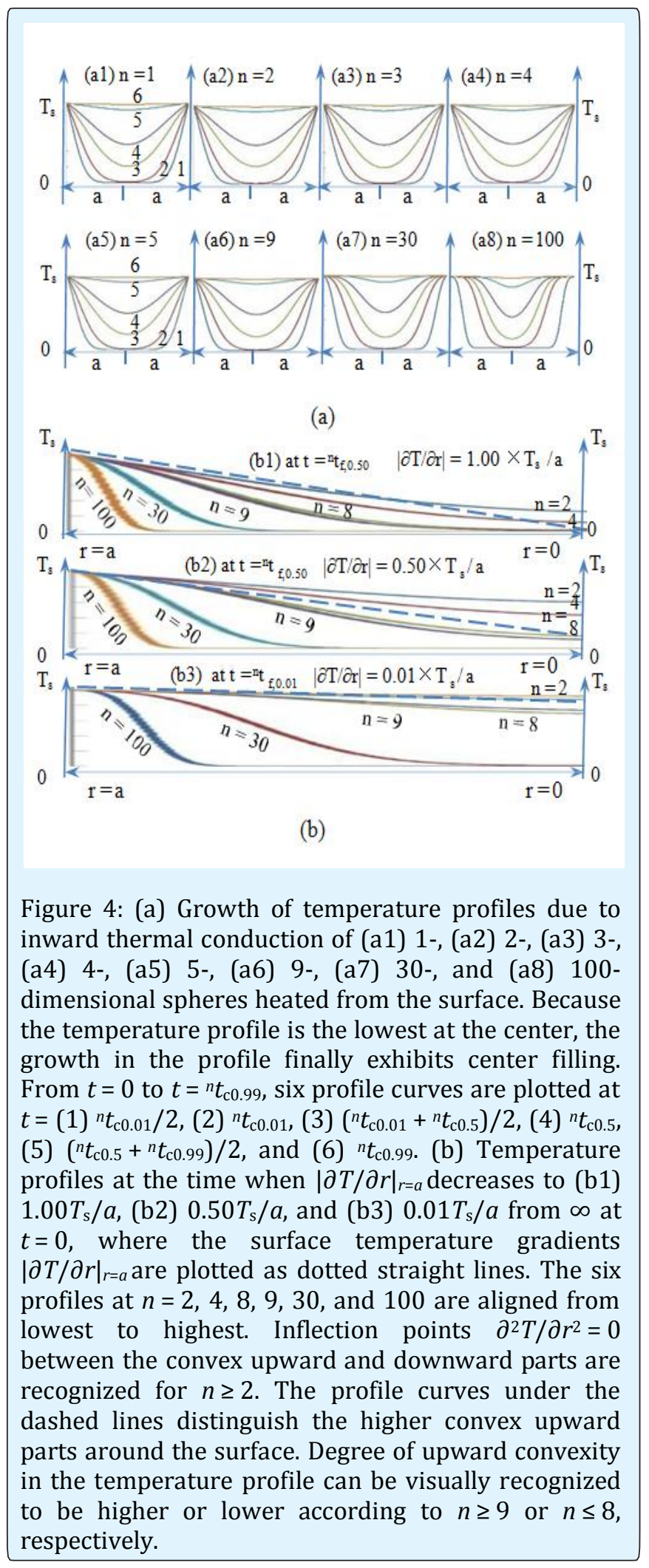

Copyright $($ C Osuga T. 


\section{Nanomedicine \& Nanotechnology Open Access}

\section{Fluid Rotation in a Sphere Described as Five- Dimensional Spherical Heating}

A rigid spherical shell with a diameter of $2 a$ is immersed in a Newtonian fluid and has the coordinate system shown in Figure 3(f). The growth of the flow in the spherical shell is represented by laminar flow layers composed of thin concentric spherical shells. When the spherical shell starts to rotate along the $z$ axis with a constant surface angular velocity of $\Omega$ at $t=0$, the spherical laminar flow layers rotate coaxially with the angular velocity $\omega(r)$ and diffuse to the center from the surface of the spherical shell. Finally, the spherical laminar flow layers approach $\Omega$. Each laminar flow layer is equivalent to the $k$-th spherical shell $\left(k=1 \rightarrow k_{\max }\right)$ with the angular velocity $\omega_{k}$ and radius $r_{k-1 / 2}$. The azimuthal angle $\theta$ is measured from the positive direction of the $z$ axis at $\theta=0$ to the negative direction of the $z$ axis at $\theta=\pi$. The $k$-th spherical shell is divided into narrow rings with a similar azimuthal angle division $\Delta \theta$, as shown in Figure $5(a)$. The ring boundaries $\theta_{j}$ are subscripted with 0 to $j_{\max }$ such that $\theta_{0}=0.0$ and $\theta_{j \max }=\pi$. The $j$-thring for $(j-1) \Delta \theta \leq \theta \leq j \Delta \theta$ in the $k$-th shell for $(k-1) \Delta r \leq r \leq k \Delta r$ is considered. The angular velocity $\omega_{j, k}$ of the ring is defined at the radial and azimuthal center of the ring, i.e., at $r=r_{k-1 / 2}$ and $\theta=\theta_{j-1 / 2}$. Because the cross section $S$ and the circumference $L$ of the ring are $S=\Delta r \times\left(r_{k-1 / 2} \Delta \theta\right)$ and $L=2 \pi r_{k-1 / 2} \times \sin \theta_{j-1 / 2}$, respectively, as shown in Figure 5, the volume of the ring $V=L S$ is expressed as $V=2 \pi r_{k-1 / 2} \times \sin \theta_{j-1 / 2} \times r_{k-1 / 2} \Delta \theta \Delta r$. Because the mass $M$ of the ring is $M=\rho V$, the moment of inertia $I_{j, k}=M\left(r_{k-1 / 2} \times \sin \theta_{j-1 / 2}\right)^{2}$, defined at the radial and azimuthal center of the ring, is given as $I_{j, k}=\rho 2 \pi r_{k}$. $1 / 2^{4} \times \sin ^{3} \theta_{j-1 / 2} \Delta \theta \Delta r$. The flow velocity $v_{k}=\sin \theta_{j-1 / 2} \times r_{k-}$ $1 / 2 \times \omega_{k}$ varies at each ring in the shell because $\theta_{j-1 / 2}$ varies at each ring. However, no torques are generated between the rings in the same spherical shell at $r=r_{k-1 / 2}$ because the angular velocity is the same for all $j\left(0 \leq j \leq j_{\max }\right)$ rings in the $k$-th spherical shell, i.e., $\omega_{j, k}=\omega_{k}$. The flow grows owing to the torque acting between radially adjacent rings in a similar manner as the rotating cylindrical shell in Equation (2.11).

Because the torque $\sigma$ acting on the $j$-th (azimuthal) ring in the $k$-th(radial) shell from the radially adjacent $j$-th ring in the $(k+1)$-th shell is expressed as the product of the length of the rotation moment $\sin \theta_{j-1 / 2} r_{k}$, the contacting surface area $\left(2 \pi \sin \theta_{j-1 / 2} r_{k}\right) \times\left(r_{k} \Delta \theta\right)$, and the velocity shear drag $\mu \Delta v_{k} / \Delta r=\mu\left(\sin \theta_{j-1 / 2} \times r_{k} \times \omega_{j, k+1}{ }^{m}-\sin \theta_{j-1 / 2} \times r_{k} \times \omega_{j, k^{m}}\right) / \Delta r$, $\sigma=\mu \sin \theta_{j-1 / 2} \times r_{k} \times 2 \pi \sin \theta_{j-1 / 2} \times r_{k} \times r_{k} \Delta \theta\left(\sin \theta_{j-1 / 2} \times r_{k} \times \omega_{j, k+1} m_{-}\right.$ $\left.\sin \theta_{j-1 / 2} \times r_{k} \times \omega_{j, k^{m}}\right) / \Delta r$. It should be noted that the flow velocity shear generated at the distance of $\sin \theta_{j-1 / 2} \times r_{k}$ from the $z$ axis originates from $\sin \theta_{j-1 / 2} \times r_{k} \times \omega_{j, k+1}{ }^{m}-$ $\sin \theta_{j-1 / 2} \times r_{k} \times \omega_{j, k^{m}}{ }^{2}$, which is the velocity difference crossing the boundary at $r=r_{k}$, because $r_{k}$ and $\omega_{j, k}$ are defined at the outer radial edge and the radial center of the ring, respectively. The total torque $N=N_{j, k}$ upper $+N_{j, k}$ lower acting on the $j$-th ring in the $k$-th shell is determined by the upper torque $N_{j, k}$ upper from the $j$-th ring in the $(k+1)$-th shell and the lower torque $N_{j, k}$ lower from the $j$-th ring in the $(k-1)$-th shell calculated at the present time step $m \Delta t$, where $N_{j, k}$ upper $=\mu \sin \theta_{j}$. $1 / 2 \times r_{k} \times 2 \pi \sin \theta_{j-1 / 2} \times r_{k} \times r_{k} \Delta \theta\left(\sin \theta_{j-1 / 2} \times r_{k} \times \omega_{j, k+1}{ }^{m}-\sin \theta_{j-}\right.$ $\left.1 / 2 \times r_{k} \times \omega_{j, k^{m}}^{m}\right) / \Delta r$, and $N_{j, k^{\text {lower }}}=\mu \sin \theta_{j-1 / 2} \times r_{k-1} \times 2 \pi \sin \theta_{j-}$ $1 / 2 \times r_{k-1} \times r_{k-1} \Delta \theta\left(\sin \theta_{j-1 / 2} \times r_{k-1} \times \omega_{j, k-1}{ }^{m}-\sin \theta_{j-1 / 2} \times r_{k-}\right.$

$\left.1 \times \omega_{j, k^{m}}\right) / \Delta r$, which are defined at the outer and inner radial edges of the ring, respectively. The angular velocity $\omega_{j, k^{m+1}}$ at the next time step is determined in a similar manner as the torque in Equations (2.11) and (2.12) as

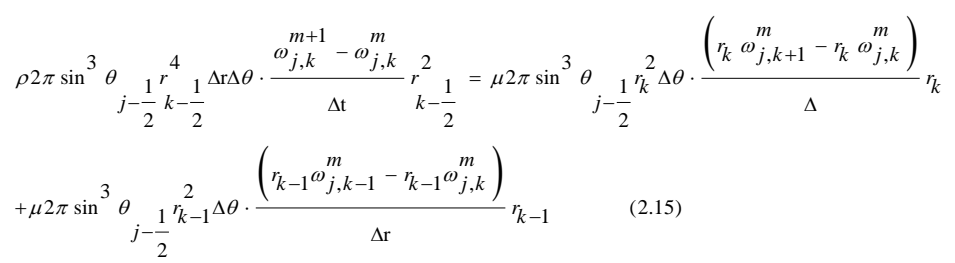

Using $r_{k}^{j}-r_{k-1}^{j} \approx j r_{k-1 / 2}^{j-1} \Delta r$, Equation (2.15) is transformed into

$\rho\left(r_{k}^{5}-r_{k-1}^{5}\right) \frac{\omega_{j, k}^{m+1}-\omega_{j, k}^{m}}{\Delta}=\mu 5 r_{k}^{4} \frac{\left(\begin{array}{c}m \\ \omega_{j, k+1}-\omega_{j, k}^{m}\end{array}\right)}{\Delta \mathrm{r}}+\mu 5 r_{k-1}^{4} \frac{\left(\begin{array}{c}m \\ \omega_{j, k-1}-\omega_{j, k}^{m}\end{array}\right)}{\Delta}$

Because time progression of $\omega_{j, k}$ for any $j$-th ring in the same $k$-th shell is described by a torque equation similar to Equation (2.16), $\omega_{j, k}$ is the same for all $j\left(0 \leq j \leq j_{\max }\right)$ rings in the $k$-th shell such that $\omega_{j, k}=\omega_{k}$. Equation (2.16) is equivalent to the thermal conduction of a fivedimensional sphere in Equation (2.3). The growth of the angular velocity profile is analogous to spherical heating from the surface for $n=5$. The exchange of $T(r)$ with $\omega(r)$ and $\chi$ with $v(=\mu / \rho)$ in Equation (2.6) for $n=5$ yields the growth shown in Figure 4(a5). The fluid between two coaxial spheres having radii of a and $b$ starts to rotate following the coaxial rotations of the inner and outer spheres, where $\omega$ is $\Omega$ and $\omega_{\text {out }}$ at $r=a$ and $b$, respectively. After a growth period, the stationary angular velocity profile is derived from Equation (2.6) for $n=5$ by setting $\partial / \partial t=0$ as [7-9].

$$
\omega=\frac{b^{3}}{\left(b^{3}-a^{3}\right)}\left[\left(\Omega-\omega_{\text {out }}\right)\left(\frac{a}{r}\right)^{3}-\Omega\left(\frac{a}{b}\right)^{3}+\omega_{\text {out }}\right]
$$




\section{Nanomedicine \& Nanotechnology Open Access}

When the sphere with a radius of $a$ rotates at $\omega=\Omega$ immersed in an infinitely wide static fluid, the angular velocity profile for $r \geq a$ is given as $\omega=(a / r)^{3} \Omega$ by letting $b / a \rightarrow \infty$ and setting $\omega_{\text {out }}=0$ in Equation (2.17). The force couple for the sphere for maintaining $\Omega$ is given as $8 \pi a^{3} \mu \Omega$ [7], which is derived from $2 \pi a \sin \theta a \Delta \theta \times a \sin \theta \times a \sin \theta\left(\partial(r \omega) /\left.\partial r\right|_{r=a}\right)$.

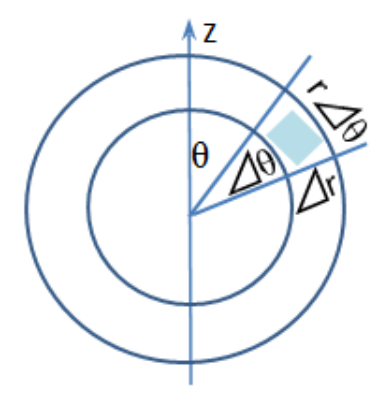

(a)

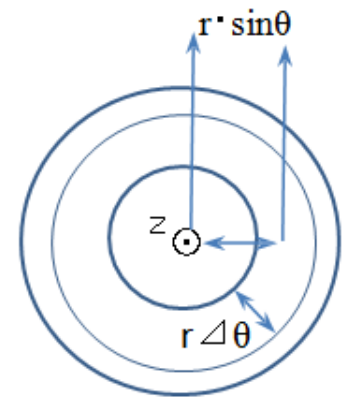

(b)
Figure 5: (a) Cross section of a ring rotating about the $z$ axis viewed perpendicular to the $z$ axis, whose crosssectional area is $r \Delta \theta \Delta r$. (b) Round circle of a ring viewed along the $z$ axis, whose width is $r \Delta \theta$.

Fluid Rotation in Two Discs Described as Slab Heating: The lamination of thin fluid discs with a radial diameter of $2 a$ and an axial thickness of $\Delta z$ along the $z$ axis constitutes a cylinder, as shown in Figure 3(e). The two surface discs at the ends of the cylinder are rigid discs located at $z= \pm h$, where symmetry with respect to $z=0$ is assumed. The discs surrounded by the two surface discs are the laminar flow layers of a Newtonian fluid, and the radial position $r$ is defined by the distance from the $z$ axis. It is assumed that no matter outside the cylinder for $r>a$ exerts action on the fluid discs for $r<a$. When the two surface discs start to rotate about the $z$ axis with a constant angular velocity $\Omega$ at $t=0$, the laminar flow layer diffuses with an angular velocity of $\omega(z)$ from the surface discs to the center at $z=0$, and the flow in the cylinder finally approaches a uniformly rotating flow with a surface angular velocity of $\Omega$. The laminar flow layers are axially divided into concentric discs indexed by $i$ $\left(i=1 \rightarrow i_{\max }\right)$ with an axial thickness of $\Delta z$. The $i$-th disc is radially divided into concentric rings indexed by $k$ $\left(k=1 \rightarrow k_{\max }\right)$ with a radial thickness of $\Delta r$, as shown in Figure 2. The $k$-th ring for $(k-1) \Delta r \leq r \leq k \Delta r$ in the $i$-th disc for $(i-1) \Delta z \leq z \leq i \Delta z$ is considered. The angular velocity $\omega_{i, k}$ of the ring is defined at the radial and axial center of the ring, i.e., at $r=r_{k-1 / 2}$ and $z=z_{i-1 / 2}$. Because the cross section $S$ and the circumference $L$ of the ring are $S=\Delta z \Delta r$ and $L=2 \pi r_{k-1 / 2}$, respectively, as shown in Figure 5 at $\theta=\pi / 2$, the volume of the ring $V=L S$ is derived as $V=2 \pi r_{k-1 / 2} \Delta r \Delta z$. Because the mass $M$ of the ring is $M=\rho V$, the moment of inertia $I_{k}=M r_{k-1 / 2^{2}}$, defined at the center of the ring, is given as $I_{k}=\rho 2 \pi r_{k-1 / 2}{ }^{3} \Delta r \Delta z$. Although the flow velocity $v_{k}=r_{k-1 / 2} \omega_{i, k}$ varies for each ring in the same disc, no torques are generated between the rings in the same disc because the angular velocity will be proven to be the same in the $i$-th disc, i.e., $\omega_{i, k}=\omega_{k}$. The flow grows owing to the rotation torque acting between axially adjacent rings according to Equation (2.11).

Because the torque $\sigma$ acting on the $k$-th (radial) ring in the $i$-th (axial) disc from the axially adjacent $i$-th ring in the $(i+1)$-th disc is expressed as the product of the length of the rotation moment $r_{k}$, the contacting surface area $2 \pi r_{k} \Delta r$, and the velocity shear drag $\mu \Delta v_{i, k} / \Delta z=\mu\left(r_{k-}\right.$ $\left.1 / 2 \omega_{i+1, k^{m}}-r_{k-1 / 2} \omega_{i, k^{m}}\right) / \Delta z, \quad \sigma=\mu r_{k-1 / 2} \times 2 \pi r_{k-1 / 2} \Delta r\left(r_{k-1 / 2} \omega_{i+1, k^{m}-}\right.$ $\left.r_{k-1 / 2} \omega_{i, k^{m}}\right) / \Delta z$. The total torque $N=N_{k}^{\text {upper }}+N_{k}^{\text {lower }}$ acting on the $k$-th ring in the $i$-th shell is determined by the upper torque $N_{i}$ upper from the $(i+1)$-th disc and the lower torque $N_{i}$ lower from the $(i-1)$-th disc calculated at the present time step $m \Delta t$, where $N_{k}^{\text {upper }}=\mu r_{k-1 / 2} \times 2 \pi r_{k \text { - }}$ $1 / 2 \Delta r\left(r_{k-1 / 2} \omega_{i+1, k^{m}}-r_{k-1 / 2} \omega_{i, k^{m}}\right) / \Delta z$, and $N_{k}^{\text {lower }}=\mu r_{k-1 / 2} \times 2 \pi r_{k-}$ $1 / 2 \Delta r\left(r_{k-1 / 2} \omega_{i-1, k^{m}}-r_{k-1 / 2} \omega_{i, k^{m}}\right) / \Delta r$. The angular velocity $\omega_{i, k^{m+1}}$ of the $k$-th ring in the $i$-th disc at the next time step $(m+1) \Delta t$ is determined according to torque equation in Equation(2.11) as

$$
\rho\left(r_{k}-r_{k-1}\right) \frac{\omega_{i, k}^{m+1}-\omega_{i, k}^{m}}{\mathrm{t}}=\mu \frac{\left(\begin{array}{c}
m \\
\omega_{i, k+1}-\omega_{i, k}^{m}
\end{array}\right)}{\mathrm{z}}+\mu \frac{\left(\begin{array}{c}
m \\
\omega_{i, k-1}-\omega_{i, k}^{m}
\end{array}\right)}{\mathrm{z}}
$$

Where $r_{k}-r_{k-1}=\Delta r$ was used. Because time progression of $\omega_{i, k}$ for any $k$-th ring in the same $i$-th disc is described by a torque equation similar to Equation (2.18), $\omega_{i, k}$ is the same for all $k\left(0 \leq k \leq k_{\max }\right)$ rings in the $i$-th disc, i.e., $\omega_{i, k}=\omega_{i}$. Equation (2.18) is equivalent to the slab heating of a volume surrounded by two parallel plates separated by $2 h$, as shown in Figure 3(b), i.e., the thermal conduction of a one-dimensional sphere in Equation (2.3). The growth of the angular velocity profile is analogous to slab heating from surface for $n=1$ and shown in Figure 4(a1). 


\section{Nanomedicine \& Nanotechnology Open Access}

\section{Specificity of the Diffusion Equation in the Geometry of an $\boldsymbol{n}$-Dimensional Sphere}

Analytical Formulae for the Heating Modes in One, Two, and Three Dimensions: Thermal conduction is restricted to the geometries of the heat sources and the surrounding volumes through which heat is transferred. The heat sources of a plane, a slab, an $n$-dimensional sphere, and a cube are shown in Figure 3 . The origin of the radial coordinate $r$ is located at the center $r=0$, and the surface of the volume is located at $r=a$. The centrosymmetric temperature profile $T(r, t)$ is measured in degrees Kelvin. The slab heating of a volume surrounded by two parallel plates separated by a distance of $2 a$ is shown in Figure 3(b). The two positive directions of the $r$ axis originate at the origin and have opposite directions. The heating from cylindrical and spherical surfaces is shown in Figures 3(e) and 3(f), respectively, where the radii of the cylinder and sphere are $a$. The thermal conduction for the slab, cylinder, and sphere corresponds to the heating modes of one $(n=1)$, two $(n=2)$, and three $(n=3)$-dimensional spheres, respectively, where $n$ is substituted into Equation (2.6). The boundary conditions are described by $r$ and the same for the three heating modes, as shown in Equation (2.7), where both the volume and surface have the uniform temperature $T(r, t)=T_{\mathrm{c}}$ before $t=0$, and the temperature at the surface is maintained at a constant value $T_{\mathrm{s}}$ after $t=0$. Consequently, the volume is heated from the surface at a constant temperature $T_{\mathrm{s}}$ for $t \geq 0$.

By applying the boundary conditions

$$
T(r \leq a, t<0)=T_{c} \text { and } T(r=a, t \geq 0)=T_{s}
$$

where $T_{\mathrm{c}}=0$ and $T_{\mathrm{s}}=1$, Equation (2.6), the growth of the temperature profiles for one, two, and three-dimensional spheres is analytically derived as [10-12].

$$
\begin{aligned}
& T(r, t)=T_{s}+2\left(T_{c}-T_{s}\right) \sum_{n=1}^{\infty} \frac{(-1)^{n-1}}{a_{n}} \cos \left(a_{n} \frac{r}{a}\right) \exp \left(-a_{n}^{2} \chi \frac{t}{a^{2}}\right), \mathrm{a}_{\mathrm{n}}=\left(\mathrm{n}-\frac{1}{2}\right) \pi \quad(3.2 a) \\
& T(r, t)=T_{s}+2\left(T_{c}-T_{s}\right) \sum_{n=1}^{\infty} \frac{J_{0}\left(b_{n} \frac{r}{a}\right)}{b_{n} J_{1}\left(b_{n}\right)} \exp \left(-b_{n}^{2} \chi \frac{t}{a^{2}}\right), \mathrm{J}_{0}\left(\mathrm{~b}_{\mathrm{n}}\right)=0 \\
& T(r, t)=T_{s}+2\left(T_{c}-T_{s}\right) \sum_{n=1}^{\infty} \frac{(-1)^{n-1}}{s_{n}}-\frac{1}{r} \sin \left(s_{n} \frac{r}{a}\right) \exp \left(-s_{n}^{2} \chi \frac{t}{a^{2}}\right), \mathrm{s}_{\mathrm{n}}=\mathrm{n} \pi
\end{aligned}
$$

respectively, where $J_{0}$ and $J_{1}$ are the zeroth- and firstorder Bessel functions, respectively; and $b_{n}(>0)$ satisfies $J_{0}\left(b_{n}\right)=0$. Because Equation (3.2) involves exponentially decaying terms, the inward thermal conduction of an $n$ dimensional sphere finally approaches a flat temperature profile throughout the volume $(t \rightarrow \infty)$, expressed as

$$
T(r \leq a, t \rightarrow \infty)=T_{S}
$$

which is obtained by solving Equation (2.6) and eliminating the time derivative $\partial / \partial \mathrm{t}$ on the left-hand side. The growing temperature profiles in Equations (3.2a), $(3.2 b)$, and $(3.2 c)$ are plotted in Figures 4(a1), 4(a2), and 4(a3), respectively, where the horizontal and vertical axes are the radial position $r$ and temperature $T$, respectively.

\section{Time Period for Increasing the Center Temperature to the Surface Temperature}

Since heat is transferred from the surface to the center, the temperature at the center is always the lowest until the end of the growth. By retaining the slowest exponential decay terms in Equation (3.2), the temperature profiles around the center at the final growth stage are given as

$$
\begin{aligned}
& T=T_{S}+2\left(T_{c}-T_{S}\right) \frac{2}{\pi} \cos \left(\frac{\pi}{2} \frac{r}{a}\right) \exp \left(-\frac{\pi^{2}}{4} \chi \frac{t}{a^{2}}\right) \\
& T=T_{S}+2\left(T_{c}-T_{S}\right) \frac{J_{0}\left(b_{1} \frac{r}{a}\right)}{b_{1} J_{1}\left(b_{1}\right)} \exp \left(-b_{1}^{2} \chi \frac{t}{a^{2}}\right), b_{1}=2.40 \\
& T=T_{S}+2\left(T_{c}-T_{S}\right) \frac{1}{\pi} \frac{1}{\pi} \sin (\pi-4.45) \\
& \pi
\end{aligned}
$$

where $b_{1}=2.4, J_{0}(0)=1, J_{0}\left(b_{1}\right)=0$, and $J_{1}\left(b_{1}\right)=0.519$. The three growth periods ${ }^{1} t_{\mathrm{g}},{ }^{2} t_{\mathrm{g}}$, and ${ }^{3} t_{\mathrm{g}}$ of the three heating modes assume that ${ }^{1} t_{\mathrm{g}}=5 \times 4 a^{2} /\left(\pi^{2} \chi\right),{ }^{2} t_{\mathrm{g}}=5 \times a^{2} /\left(2.4{ }^{2} \chi\right)$, and ${ }^{3} t_{\mathrm{g}}=5 \times a^{2} /\left(\pi^{2} \chi\right)^{3}$. When $t>{ }^{1} t_{\mathrm{g}},{ }^{2} t_{\mathrm{g}}$, and ${ }^{3} t_{\mathrm{g}}$, the slowest exponential decay terms in the three heating modes in Equations (3.4a), (3.4b), and (3.4c) become less than 0.0067 , where $\exp (-5)=0.0067$ is used. Thus, ${ }^{1} t_{\mathrm{g}}$, ${ }^{2} t_{\mathrm{g}}$, and ${ }^{3} t_{\mathrm{g}}$ originating from the slowest exponential decay terms are the simplest evaluation of the growth period. In contrast, the growth period ${ }^{n} t_{c, f}$ is defined as the time when the temperature at the center $T_{0}=T(r=0)$ is 


\section{Nanomedicine \& Nanotechnology Open Access}

$f(0.0 \leq f \leq 1.0)$ multiplied by the surface temperature $T_{\mathrm{s}}$, where $T_{\mathrm{c}}=0$, as

$$
{ }_{t_{c, f}}=\frac{4}{\pi^{2}} \frac{a^{2}}{\chi} \log _{e} \frac{4}{\pi(1-f)},{ }^{2} t_{c, f}=\frac{1}{2.4^{2}} \frac{a^{2}}{\chi} \log _{e} \frac{2}{b_{1} J_{1}\left(b_{1}\right)(1-f)},{ }^{3} t_{c, f}=\frac{1}{\pi^{2}} \frac{a^{2}}{\chi} \log _{e} \frac{4}{\pi(1-f)}
$$

By setting $f=0.99$ in Equation (3.5), ${ }^{n} t_{c, 0.99}$ could be obtained analytically for $n=1,2$, and 3, where ${ }^{1} t_{c, 0.99},{ }^{2} t_{c, 0.99}$, and ${ }^{3} t_{c, 0.99}$ slightly deviate from ${ }^{1} t_{\mathrm{g}},{ }^{2} t_{\mathrm{g}}$, and ${ }^{3} t_{\mathrm{g}}$, respectively. ${ }^{n} t_{\mathrm{g}}$ and ${ }^{n} t_{\mathrm{c}, 0.99}$ are compared as follows:

$$
\begin{aligned}
& 1_{t_{g}=2.03 \frac{a^{2}}{\chi}}\left[=\frac{20}{\pi^{2}} \frac{a^{2}}{\chi}\right],{ }^{2} t_{g}=0.868 \frac{a^{2}}{\chi}\left[=\frac{5}{2.4^{2}} \frac{a^{2}}{\chi}\right],{ }^{3} t_{g}=0.507 \frac{a^{2}}{\chi}\left[=\frac{5}{\pi^{2}} \frac{a^{2}}{\chi}\right](3.6 a) \\
& 1_{t_{c, 0.99}}=1.96 \frac{a^{2}}{\chi}\left[=\frac{4}{\pi^{2}} \frac{a^{2}}{\chi} \log _{e} \frac{400}{\pi}\right],{ }^{2} t_{c, 0.99}=0.882 \frac{a^{2}}{\chi}\left[=\frac{1}{2.4^{2}} \frac{a^{2}}{\chi} \log _{e} \frac{200}{b_{1} J_{1}\left(b_{1}\right)}\right],{ }^{3} t_{c, 0.99}=0.490 \frac{a^{2}}{\chi}\left[=\frac{1}{\pi^{2}} \frac{a^{2}}{\chi} \log _{e} \frac{400}{\pi}\right](3.6 b)
\end{aligned}
$$

The steady flat temperature profiles for one-, two-, and three dimensional spheres are completed after $t={ }^{1} t_{c, 0.99}$, ${ }^{2} t_{c, 0.99}$, and ${ }^{3} t_{c, 0.99}$, respectively.

\section{$n$-Dimensional Cubes Inscribed within and} Circumscribing an $n$-Dimensional Sphere : Although there are no analytical solutions for the inward thermal conduction of $n$-dimensional spheres heated from the surface for $n \geq 4$, the growth period can be estimated through an extension of slab heating (one-dimension) to $n$ dimensions, i.e., the thermal conduction of an $n$ dimensional cube (hypercube) heated from the surface. A cube and its three-dimensional orthogonal coordinate system $r_{1}, r_{2}$, and $r_{3}$ perpendicular to three planes are shown in Figure 3(d). The origins of $r_{1}, r_{2}$, and $r_{3}$ are located at the center of the cube, and the radius $a$ of the cube is defined by the distance between the center and the plane. One- and two-dimensional cubes are a slab and square duct, as shown in Figures 3(b) and 3(c), respectively. In a similar manner, an $n$-dimensional cube described by the $n$-dimensional orthogonal coordinates $r_{1}$, $r_{2}, \ldots, r_{n-1}, r_{n}$ is defined. The temperature $T$ inside the $n$ dimensional cube is expressed as $T\left(r_{1}, r_{2}, \ldots, r_{n-1}, r_{n}, t\right)$. The thermal conduction equation for the $n$-dimensional cube is an extension of Equation (2.8):

$$
\frac{\partial T}{\partial t}=\chi\left(\frac{\partial^{2} T}{\partial r_{1}^{2}}+\frac{\partial^{2} T}{\partial r_{2}^{2}}+\cdots---\frac{\partial^{2} T}{\partial r_{n-1}^{2}}+\frac{\partial^{2} T}{\partial r_{n}^{2}}\right)
$$

The boundary conditions are similar to Equation (3.1) because $r_{1}, r_{2}, \ldots, r_{n-1}, r_{n}$ are equivalent to $r$. Moreover, the thermal conduction in a one-dimensional cube is similar to the slab heating in Equation (3.2a). The solution of Equation (3.7) is an extension of Equation (3.2a), which is a product of $n$ Fourier cosine functions of $r_{1}, \ldots, r_{n}$, of which the exponential decay term involves $n$. The temperature profile $T\left(r_{1}, r_{2},----, r_{n-1}, r_{n}, t\right)$ around the center at the final growth stage is derived as an extension of the slab heating in Equation (3.4a) to $n$ dimensions as

$$
T=T_{s}+2\left(T_{c}-T_{s}\right)\left(\frac{2}{\pi}\right)^{n} \exp \left(-\frac{\pi^{2}}{4} \mathrm{n} \chi \frac{t}{a^{2}}\right)
$$

The distance between two facing planes and the length of the diagonal are $2 a$ and $2 a \sqrt{n}$, respectively, for the $n$ dimensional cube. The relations between the cubes circumscribing and inscribed within a sphere with a radius of $a$ are shown in Figure $1(b)$, where the radii of the sphere and circumscribed cube are $a$ and that of the inscribed cube is $a / \sqrt{3}$. In a similar manner, the radii $a_{\text {circ }}$ and $a_{\text {insc }}$ of the $n$-dimensional cube circumscribing and inscribed within an $n$-dimensional sphere with a radius of $a$ are determined to be $a_{\text {circ }}=a$ and $a_{\text {insc }}=a / n$, respectively. $a_{\text {circ }}$ and $a_{\text {insc }}$ are substituted for $a$ in Equation(3.8) to evaluate the growth periods ${ }^{n} t_{\text {circ }}$ and ${ }^{n} t_{\text {insc }}$ for the inward thermal conduction of the circumscribed and inscribed $n$-dimensional cubes by 


\section{Nanomedicine \& Nanotechnology Open Access}

retaining the slowest exponential decay terms in a similar manner as the derivation of ${ }^{1} t_{\mathrm{g}}$ in Equation(3.6a) as

$$
n_{t_{\text {circ }}}=\frac{1}{n} \frac{20}{\pi^{2}} \frac{a^{2}}{\chi} \text { and } n_{t_{\text {insc }}}=\frac{1}{n^{2}} \frac{20}{\pi^{2}} \frac{a^{2}}{\chi}, n \geq 2
$$

The growth period ${ }^{n} t_{\mathrm{g}}$ of the thermal conduction of the $n$ dimensional sphere heated from the surface is shorter and longer than those of the $n$-dimensional circumscribed and inscribed cubes, respectively. Therefore, the range of ${ }^{n} t_{\mathrm{g}}$ of an $n$-dimensional sphere is determined according to the inequality ${ }^{n} t_{\text {insc }} \leq{ }^{n} t_{\mathrm{g}} \leq{ }^{n} t_{\text {circ. }}$ Using Equation (3.9), the range of ${ }^{n} t_{\mathrm{g}}$ is expressed in terms of the power of $n$ as

$$
\frac{1}{n^{2}} \frac{20}{\pi^{2}} \frac{a^{2}}{\chi}<{ }^{n} t_{g}<\frac{1}{n} \frac{20}{\pi^{2}} \frac{a^{2}}{\chi}, n \geq 2
$$

The growth periods evaluated from the extension of the slab heating in Equation (3.2a) by retaining the slowest exponential decay terms in Equations (3.4a) and (3.6a) lack rigorous precision, especially for higher values of $n$ because the temperature profile at the final growth stage should be calculated using the products of the lower and higher exponential decay terms in higherdimensional cubes, in contrast to the slab heating calculated using only the lowest decay term in Equation (3.4a). However, the dependence of the growth period on $n$ can be estimated on the basis of the inequality in Equation (3.10). Because ${ }^{2} t_{\text {insc }}=0.508 a^{2} / \chi,{ }^{2} t_{\text {circ }}=1.02 a^{2} / \chi$ and ${ }^{3} t_{\text {insc }}=0.226 a^{2} / \chi,{ }^{3} t_{\text {circ }}=0.677 a^{2} / \chi$ in Equation(3.9), the analytically derived growth periods ${ }^{2} t_{c, 0.99}=0.882 a^{2} / \chi$ and ${ }^{3} t_{c, 0.99}=0.490 a^{2} / \chi$ for two- and three-dimensional spheres, respectively, as shown in Equation (3.6b), were found to be included in the inequality in Equation (3.10). Although the dependence of the growth period on $n$ can be quantitatively estimated from the fact that the surfacearea-to-volume ratio $n S / n V$ of the $n$-dimensional sphere is proportional to $n / a$ from Equation (2.1), the qualitative evaluation of the dependence is given by Equation (3.10).

\section{Growth of Convex Upward and Downward Temperature Profiles}

\section{Numerical Accuracy Satisfying von Newman $n$ and Rightmyer's Analysis and the CFL Condition}

A numerical simulation of the thermal conduction can be performed using the difference scheme in Equation
(2.4) and the boundary conditions in Equation (3.1) with $\chi=1$ and $a=1$. During the simulation, $T_{k}{ }^{m+1}$ shifts to $T_{k}{ }^{m}$ at the next time step. The thermal conduction equation in Equation (2.6) has both a diffusion term $\partial^{2} T / \partial r^{2}$, as shown in Equation (2.8), and an advection term $\partial T / \partial r$, as shown in Equation (2.10) for $n \geq 2$. The diffusion equation in Equation (2.8) is restricted to the following stable simulation condition obtained by a von Neumann stability analysis $[13,14]$ :

$$
t<\frac{(r)^{2}}{2 x}
$$

The hyperbolic equation in Equation (2.10) is restricted to the CFL condition $[15,16]$. The CFL condition is expressed as $|u|<\Delta r / \Delta t$; that is, the heat transport velocity $|u|=\chi(n-1) / r$ appearing in the spherical thermal conduction equation should be less than the numerical grid velocity $\Delta r / \Delta t$ in the hyperbolic equation in Equation (2.10). Because $u$ is proportional to $r^{-1},|u|$ takes its maximum at $r=\Delta r$, which is the outer radius of the innermost shell. Thus, the CFL condition in Equation (2.10) should adopt $|u|$ as the maximum value of $|u|=\chi$ $(n-1) / \Delta r$ and is expressed as

$$
t<\frac{(r)^{2}}{(n-1) \chi}
$$

Thus, $\Delta t$ for simulating Equation (2.10) should decrease as $n$ is increased, as shown in Equation (4.2), in contrast to $\Delta t$ for simulating Equation (2.8), which indicates no change with the increase in $n$, as shown in Equation (4.1). $\Delta t$ for simulating the planar diffusion equation in Equation (2.8) should satisfy only Equation (4.1), whereas that for the spherical diffusion equation in Equation (2.6) should satisfy both Equations (4.1) and (4.2). Therefore, the intersection between the two inequalities in Equations (4.1) and (4.2) is expressed as

$$
t<\frac{(r)^{2}}{\xi \chi}, \xi=A+B(n-1), A \geq 2.0 \text { and } B \geq 1.0
$$

Equation (4.3) approaches Equations (4.1) and (4.2) as $n$ becomes low and high, respectively. The coefficients $A$ and $B$ in Equation (4.3) are derived from the stability condition in Equation (4.1) for the diffusion term in 


\section{Nanomedicine \& Nanotechnology Open Access}

Equation (2.8) and that of Equation (4.2) for the advection term in Equation (2.10), respectively.

Even though Equation (4.3) is satisfied, the numerical accuracy should be evaluated from the balance of the heat influx entering at the surface and the increase in the heat energy of the entire volume. The heat influx $\Gamma_{\mathrm{h}}$ is proportional to the temperature gradient at the surface $|\partial T / \partial r|_{r=a}$ and the surface area ${ }^{n} S$, which is expressed as $\Gamma_{\mathrm{h}}=\lambda^{n} S|\partial T / \partial r|_{r=a}$ and measured in joules per second. Within $\Delta t$ at the $m$-th time step, $\Gamma_{\mathrm{h}}$ and the heat increase in the entire volume of the sphere are balanced and expressed on the left- and right-hand sides, respectively, of the following equation:

$$
\lambda^{n} S \Delta t \frac{T_{\max }^{m}-T_{\max }^{m}-1}{\Delta r}=\rho C_{p} \sum_{k=1}^{\max } n V_{k}\left(T_{k}^{m}-T_{k}^{m-1}\right)
$$

where the superscript $m$, subscript $k$, and ${ }^{n} V_{k}$ are the $m$-th time step, the $k$-th spherical shell, and the volume of the $k$ th spherical shell, respectively. In Equation (4.4), $\left(T_{\max }{ }^{m}-\right.$ $\left.T_{\max -1^{m}}\right) / \Delta r$ is $|\partial T / \partial r|_{r=a}$, and both sides have units of joules. Summing both sides of Equation (4.4) from $m=1$ to $m=m$ leads to

$$
\lambda^{n} S \Delta t \sum_{m=1}^{m} \frac{T_{\max }^{m}-T_{\max -1}^{m}}{\Delta r}=\rho C_{p} \sum_{k=1}^{k_{\max }}\left({ }^{n} V_{k} T_{k}^{m}-{ }^{n} V_{k} T_{k}^{1}\right)
$$

If the time steps of 1 and $m+1$ are the initial and final states, respectively, $T_{k}{ }^{1}$ and $T_{k}{ }^{m+1}$ are 0 and 1 , respectively. Thermal conduction starts at $t=0$. The period from $t=0$ until $t={ }^{n} t_{c, 0.99}$, defined as the time when the temperature at the center of the $n$-dimension sphere reaches 0.99 of the surface temperature, is the full profile completion period, which will be discussed in the next section. The summation on the left-hand side of Equation (4.5) should be continued at each time step from $t=0$ until $t={ }^{n} t_{c, 0.99}$. After $t={ }^{n} t_{c, 0.99}$, the right-hand side of Equation (4.5) becomes $\rho C_{\mathrm{p}}{ }^{n} V$ because $T_{k}^{m}\left(k=1 \rightarrow k_{\max }\right)$ is 1.0 and $\sum^{n} V_{k}={ }^{n} V$, as derived from Equation (2.2). The residual ${ }^{n} R_{c, 0.99}$ calculated from the difference between the left- and right-hand sides of Equation (4.5) at $t={ }^{n} t_{c, 0.99}$ determines the numerical accuracy:

$$
n_{R_{c, 0.99}}=\frac{n \chi t \sum_{m=1}^{m} \frac{T_{\max }^{m}-T_{\max -1}^{m}-a}{r}}{a}
$$

Where ${ }^{n} S /{ }^{n} V=n / a$, derived from Equation (2.1), and $\chi=\lambda /\left(\rho C_{\mathrm{v}}\right)$ are used. A simulation result with a lower or higher $\left|{ }^{n} R_{c, 0.99}\right|$ indicates a higher or lower numerical accuracy, respectively.

Figure 6(a) shows the dependence of $\xi$ in Equation (4.3) on $A, B$, and $n$, where the horizontal and vertical axes are $\log _{10} n$ and $\xi$, respectively. There are four curves (1), (2), (3), and (4) with various values of $A$ and $B$ used in Equation (4.3): (1) $A=10.0, B=1.0$; (2) $A=2.0, B=1.0$; (3) $A=2.0, B=0.50$; and (4) $A=2.0, B=0.25$. With the shell thickness $\Delta r=a / 500$, the dependence of ${ }^{n} R_{c, 0.99}$ in Equation (4.6) on $n$ is shown in Figure 6(b), where the horizontal and vertical axes are $\log _{10}(n)$ and ${ }^{n} R_{c, 0.99}$ ranging from $-7.0 \times 10^{-3}$ to $+1.0 \times 10^{-3}$, respectively. The four curves (1) $\rightarrow$ (4) adopt the same values of $A$ and $B$ in Figures 6(a) and (b). A higher $A$ and $B$ results in a higher $\xi$ in the upper part of Figure 6(a). Because $\Delta t$ is inversely proportional to $\xi$, as shown in Equation (4.3), a higher $\xi$ yields a lower $\Delta t$ and a higher accuracy with a lower $\left|{ }^{n} R_{c, 0.99}\right|$. Because analytical solutions have been obtained for $n \leq 3$, adequate values of $A$ and $B$ should be determined in order to restrict $\left|{ }^{n} R_{\mathrm{c}, 0.99}\right|$ within $10^{-3}$ in the simulation for $n \geq 4$. When we adopt curve (2) $(A=2.0$ and $B=1.0)$ in Figure 6, $\left|{ }^{n} R_{c, 0.99}\right|$ is restricted within $1.0 \times 10^{-3}$ from $n=5$ to $n=100$, while $\left|{ }^{n} R_{c, 0.99}\right|=1.4 \times 10^{-3}$ at $n=4$. When we adopt curve (1) $(A=10.0$ and $B=1.0)$, the range of $\left|{ }^{n} R_{c, 0.99}\right|$ is restricted within $1.0 \times 10^{-3}$ from $n=4$ to $n=100$. Therefore, simulations from $n=4-100$ will be conducted with $\Delta r=a / 500, A=10.0$, and $B=1.0$ [curve (1)] hereafter because $\left|{ }^{n} R_{c, 0.99}\right|$ is restricted within $10^{-3}$ for $4 \leq n \leq 100$.

The lower $\Delta t$ created by $\xi$ in the upper part of Figure 6(a) yields a lower $\left|{ }^{n} R_{c, 0.99}\right|$ in Figure 6(b). Both of the stable simulation conditions in Equations (4.1) and (4.2) do not hold when $A<2.0$ and $B<1.0$. Thus, simulations conducted with $\xi$ existing below curve (2) $(A=2.0$ and $B=1.0)$ in Figure 6(a) lead to a lower accuracy. The adoption of a lower $B$, as provided by curves (3) and (4) in Figure 6(a), results in a higher $\left|{ }^{n} R_{c, 0.99}\right|$ for $n \geq 4$ in Figure 6(b). Because curve (1) $(A=10.0$ and $B=1.00)$ is above curve (2) $(A=2.0$ and $B=1.0)$ in Figure $6(\mathrm{a}),{ }^{n} R_{\mathrm{c}, 0.99} \mid$ is reduced to $0.8 \times 10^{-3}$ on curve (1) for $n=4$ compared to $1.4 \times 10^{-3}$ on curve (2) for $n=4$ in Figure 1(b). A decrease in $B$ compared to $B=1.0$ yields a higher $\left|{ }^{n} R_{\mathrm{c}, 0.99}\right|$ for $n \geq 4$, as shown in Figure 6(b). Because $\xi$ on curves (3) $(A=2.0$ and $B=0.50)$ and (4) $(A=2.0$ and $B=0.25)$ exists below curve (2) in Figure 6(a), $\left|{ }^{n} R_{c, 0.99}\right|$ on curves (3) and (4) exceeds $10^{-3}$ for $1 \leq n \leq 100$, as shown in Figure 6(b) while $\left|{ }^{n} R_{c, 0.99}\right|$ is restricted within $1.4 \times 10^{-3}$ for $4 \leq n \leq 100$ on 


\section{Nanomedicine \& Nanotechnology Open Access}

curves (1) and (2). Because Equation (2.1) shows ${ }^{n} S /{ }^{n} V=n / a$, the heat transfer from the surface is more efficient for a higher $n$. As a result, the full profile completion period ${ }^{n} t_{c, 0.99}$ becomes longer, and a larger number of time steps is required to complete the simulation for a lower $n$. Thus, $\left|{ }^{n} R_{c, 0.99}\right|$ exceeds $10^{-3}$ for $n \leq 3$, even for the adoption of the lowest $\Delta t$ on curve (1) $(A=10.0$ and $B=1.00)$. The higher $\left|{ }^{n} R_{c, 0.99}\right|$ for $n \leq 3$ causes no problems because numerical simulations should be conducted for $n>3$.

Simulations using $\Delta t$, where the CFL condition in Equation (4.2) does not hold, have been reported to yield meaningless divergent results $[15,16]$. Current simulations based on the conditions on curves (3) $(A=2.0$ and $B=0.50)$ and (4) $(A=2.0$ and $B=0.25)$, where CFL condition does not hold, yielded convergent results, and $\left|{ }^{n} R_{c, 0.99}\right|$ on curves (3) and (4) in Figure 6(b) is comparatively greater than those on curves (1) and (2). The region where $|u|=\chi(n-1) / r$ exceeds $\Delta r / \Delta t$, i.e., the CFL condition in Equation (4.2) does not hold, exists within a limited narrow volume near the center where $r / a<<1$. Because $\left|{ }^{n} R_{c, 0.99}\right|$ is counted from the entire volume, the simulation was estimated to yield a convergent result, which $\left|{ }^{n} R_{c, 0.99}\right|$ is restricted within $10^{-2}$, as shown in Figure 6(b), even though the condition $B<1$ on curves (3) and (4) are adopted. However, the current simulations with $A<2.0$ and $B=0$, where both Equations (4.1) and (4.2) do not hold, definitely yielded meaningless divergent results because the stable simulation condition in Equation (4.1) should hold in the entire volume of the $n$-dimensional sphere, even though CFL condition does not hold only near the center.

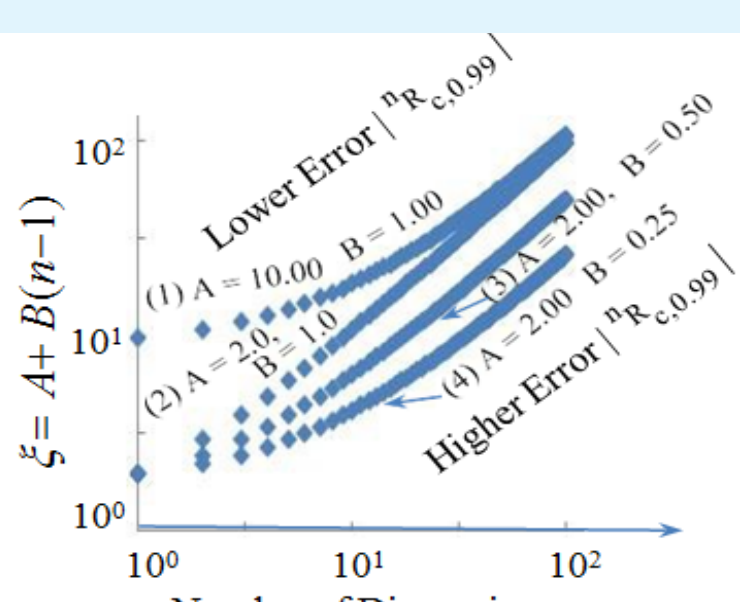

Number of Dimensions $n$

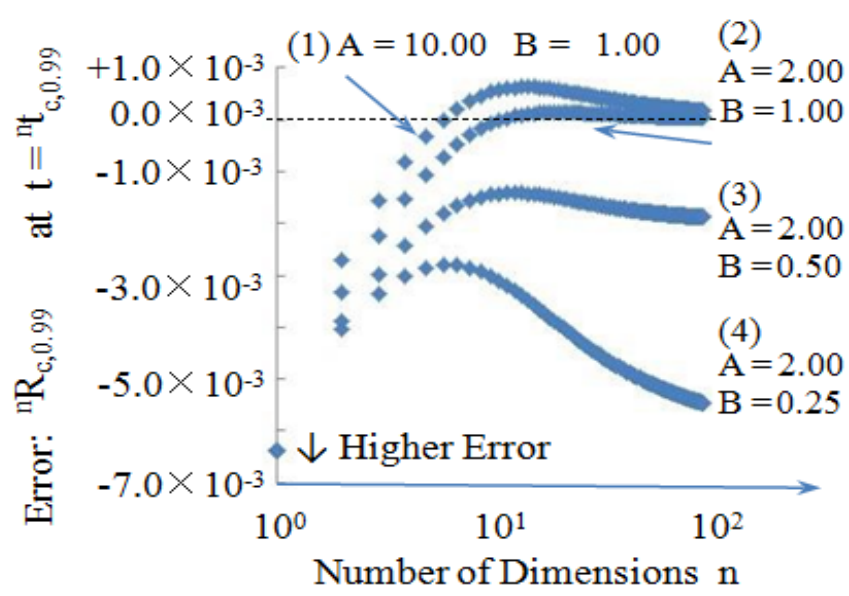

(b)

Figure 6: In the numerical simulation of spherical thermal conduction, the time step $\Delta t$ and radial division $\Delta r$ should be restricted using the relation $\Delta t<(\Delta r)^{2} /(\chi \xi)$, where $\xi$ is determined by the two parameters $A$ and $B$ as $\xi=A+B(n-$ 1). (a) Relation between $n$ and $\xi$ for (1) $A=10.0, B=1.0$; (2) $A=2.0, B=1.0$; (3) $A=2.0, B=0.50$; and (4) $A=2.0$, $B=0.25$. (b) Accuracy of the numerical simulation, as determined by the residual ${ }^{n} R_{c, 0.99}$ defined by the difference between the total heat influx at the surface and the increase of the inner heat energy summed until the full completion period $t={ }^{n} t_{\mathrm{c}, 0.99}$. The residual $\left|{ }^{n} R_{\mathrm{c}, 0.99}\right|$ remains within $10^{-3}$ for curves (1) and (2) $(A \geq 2.0$ and $B \geq 1.0)$.

\section{Initial Surface Influx Period and Final Center Filling Period}

The temperature profile grows from the surface and finally completes a stationary flat temperature profile. The heat influx is proportional to the temperature gradient at surface $|\partial T / \partial r|_{r=a}$, which is higher in the initial growth stage. Because the temperature $T_{0}$ at the center $r=0$ is always the lowest, as shown in Figure 4, the final growth stage has a center filling period during which the center temperature increases to the surface temperature level. Thus, the growth of the profile is divided into the 


\section{Nanomedicine \& Nanotechnology Open Access}

initial high surface influx stage and the final center filling stage, which are discriminated by the magnitude of $|\partial T / \partial r|_{r=a}$ and $\Delta T_{\mathrm{s}-\mathrm{c}}=\left(T_{\mathrm{s}}-T_{\mathrm{o}}\right) / T_{\mathrm{s},}$, where $T_{\mathrm{s}}-T_{\mathrm{o}}$ is the difference in the temperatures at the surface and center. Three center filling periods ${ }^{n} t_{c, 0.99},{ }^{n} t_{c, 0.50}$ and ${ }^{n} t_{c, 0.01}$ were defined as the times when $T_{\mathrm{o}}$ reaches $0.99 T_{\mathrm{s}}, 0.50 T_{\mathrm{s}}$, and $0.01 T_{\mathrm{s}}$, respectively. ${ }^{n} t_{c, 0.99},{ }^{n} t_{\mathrm{c}, 0.50}$, and ${ }^{n} t_{\mathrm{c}, 0.01}$ were found to decrease with $n$, as shown in Figure 7(a), where the horizontal and vertical axes are $\log _{10}(n)$ and $\log _{10}\left({ }^{n} t_{c, 0.99}\right)$, $\log _{10}\left({ }^{n} t_{c, 0.50}\right)$, and $\log _{10}\left({ }^{n} t_{c, 0.01}\right)$, respectively. Setting $a=\chi=1$, the center filling periods ${ }^{1} t_{c, 0.99}=1.96$, ${ }^{2} t_{c, 0.99}=0.882$, and ${ }^{3} t_{c, 0.99}=0.490$ are analytically determined from Equation (3.5) with $f=0.99$. Equation (3.5) with $f=0.50$ yielded ${ }^{1} t_{c, 0.50}=0.379,{ }^{2} t_{c, 0.50}=0.203$, and ${ }^{3} t_{c, 0.50}=0.0947$, and that with $f=0.01$ yielded ${ }^{1} t_{c, 0.01}=0.102,{ }^{2} t_{c, 0.01}=0.0840$, and ${ }^{3} t_{c, 0.01}=0.025 .{ }^{n} t_{c, 0.99}$, ${ }^{n} t_{c, 0.50}$, and ${ }^{n} t_{c, 0.01}$ for $n \leq 3$ are involved in the numerical simulation from $n=1$ to $n=100$ in Figure 7(a). Because the final steady flat profile is almost completed at the center after $t={ }^{n} t_{c, 0.99}$ at each $n,{ }^{n} t_{c, 0.99}$ is defined as the full profile completion period, and all time scales will be normalized by ${ }^{n} t_{c, 0.99}$ at each $n$ hereafter. The growth of the temperature profile of (a) 1-, (b) 2-, (c ) 3-, (d) 4-, (e ) 5-, (f) 9-, (g) 30-, and (h) 100-dimensional spheres are shown in Figure 4. During the full completion period from $t=0$ to $t={ }^{n} t_{\mathrm{c} 0.99}$, the profile curves are displayed at the following six times: $t=$ (1) ${ }^{n} t_{\mathrm{c} 0.01} / 2$, (2) ${ }^{n} t_{\mathrm{c} 0.01}$, (3) $\left({ }^{n} t_{\mathrm{c} 0.01}+{ }^{n} t_{\mathrm{c} 0.50}\right) / 2$, (4) ${ }^{n} t_{c 0.50}$, (5) $\left({ }^{n} t_{c 0.50}+{ }^{n} t_{c 0.99}\right) / 2$, and (6) ${ }^{n} t_{c 0.99}$. The decreases in ${ }^{n} t_{c, 0.99},{ }^{n} t_{c, 0.50}$, and ${ }^{n} t_{c, 0.01}$ with the increase in $n$, in comparison with planar diffusion at $n=1$, are due to the effects of the spherical geometry because the surfaceto-volume ratio is proportional to $n\left({ }^{n} S /{ }^{n} V=n / a\right)$. Figure 4 shows $\Delta T_{\mathrm{s}-\mathrm{c}}=\left(T_{\mathrm{s}}-T_{\mathrm{o}}\right) / T_{\mathrm{s}}$ decreases with time. The degree of the center filling is numerically evaluated by $\Delta T_{s-c \text {. For }}$ $n=1,10,50$, and 100 , the changes in $\Delta T_{\mathrm{s}-\mathrm{c}}$ with time are compared in Figure 7(b), where the horizontal and vertical axes are the time normalized by ${ }^{n} t_{c, 0.99}$ and $\log _{10}\left(\Delta T_{\mathrm{s}-\mathrm{c}}\right)$, respectively. The analytical solutions of Equation (3.4) indicate that $\Delta T_{\mathrm{s}-\mathrm{c}}$ decreases exponentially with time for $n \leq 3$. Although $\Delta T_{\mathrm{s}-\mathrm{c}}$ for $n=1$ decreases exponentially with time (a linear curve), it is a convex upward curve for $n \geq 2$, and the curvature is visually recognized to be distinct for $n \geq 9$. Thus, the center filling period was considered to be biased to the final stage in the full profile completion period ${ }^{n} t_{c, 0.99}$ for $n \geq 9$.

The bias of the center filling period to the final stage is evaluated by the ratios ${ }^{n} t_{c, 0.50} /{ }^{n} t_{c, 0.99}$ and ${ }^{n} t_{c, 0.01} /{ }^{n} t_{c, 0.99}$, which are plotted as a function of $n$ in Figure 7(c), where the horizontal and vertical axes are $\log _{10}(n)$ and
${ }^{n} t_{c, 0.50} /{ }^{n} t_{c, 0.99}$ and ${ }^{n} t_{c, 0.01} /{ }^{n} t_{c, 0.99}$, respectively. These two ratios were found to approach asymptotic lines for $n \geq 9$ and show that the center filling period is biased to the final stage with the increase of $n$. The time for $T_{0}$ to reach $0.50 T_{\mathrm{s}}$ requires $70 \%$ of the full profile completion period ${ }^{n} t_{c, 0.99}$ at $n=100$, whereas that requires $20 \%$ of ${ }^{n} t_{c, 0.99}$ at $n=1$. The time for $T_{\mathrm{o}}$ to reach $0.01 T_{\mathrm{s}}$ requires $50 \%$ of ${ }^{n} t_{c, 0.99}$ at $n=100$, while that requires only $2 \%$ of ${ }^{n} t_{c, 0.99}$ at $n=1$. Thus, the center filling period was determined to be biased to the final stage with the increase in $n$. Because there is a linear relation between $\log _{10}(n)$ and $\log _{10}\left({ }^{n} t_{c, 0.99}\right)$ in Figure 7(a), ${ }^{n} t_{c, 0.99}$ is expected to be proportional to $n$ to the power index of ${ }^{n} \delta_{c, 0.99}$. Because ${ }^{n} \delta_{c, 0.99}$ was calculated from $\quad{ }^{n} t_{c, 0.99} /{ }^{n-1} t_{c, 0.99}=n^{\delta} /(n-1)^{\delta}, \quad$ i.e., ${ }^{n} \delta_{c, 0.99}=\log _{10}\left({ }^{n} t_{c, 0.99} /{ }^{n-1} t_{c, 0.99}\right) / \log _{10}\{n /(n-1)\}, \quad{ }^{n} \delta_{c, 0.99}$ cannot be defined for one dimension. The dependence of ${ }^{n} \delta_{c, 0.99}$ on $n$ is plotted in Figure 7(d), where the horizontal and vertical axes are $\log _{10}(n)$ and ${ }^{n} \delta_{c, 0.99}$, respectively. The power indices ${ }^{2} \delta_{c, 0.99}=-1.159$ and ${ }^{3} \delta_{c, 0.99}=-1.213$ are calculated from the analytical solutions of Equation (3.4) and are in agreement with the numerical results. Because the rate of change in $\left|{ }^{n} \delta_{c, 0.99}\right|$ remains within $12 \%$ from $n=2$ to $n=100$, a power law was determined, wherein ${ }^{n} t_{c, 0.99}$ was proportional to $n$ to the power of ${ }^{n} \delta_{c, 0.99}$. The numerically determined values of ${ }^{4} \delta_{c, 0.99}$ and ${ }^{5} \delta_{c, 0.99}$ are 1.241 and -1.259 , respectively. The power index ${ }^{n} \delta_{c, 0.99}$ was assumed to be -1.25 , which is the average of ${ }^{4} \delta_{c, 0.99}$ and ${ }^{5} \delta_{c, 0.99}$. Therefore, ${ }^{n} t_{c, 0.99}$ affected by the spherical geometry was found to be expressed as a power law in Equation( $4.7 a$ ). The reasons to adopt the average of ${ }^{4} \delta_{c, 0.99}$ and ${ }^{5} \delta_{c, 0.99}$ are as follows. The average value is almost the center value from $n=1$ to $n=100$, the growth periods for $n=4$ and 5 can be verified through a fluid rotation measurement, and the average of the two values has more reproducibility than a least-squares fitting. In a previous study, ${ }^{n} \delta_{c, 0.99}$ was assumed to be -1.24 from a least-squares fitting from $n=1$ to 100 [12]. Because the surface-to-volume ratio increases with $n$, the influx efficiency increases with $n$. Thus, another relation between ${ }^{n} t_{c, 0.99}$ and $n$ can be estimated, as expressed in Equation $(4.7 b)$. Because it is expected that the power index ${ }^{n} \delta_{c, 0.99}$ is in the range from -2.0 to -1.0 for the thermal conduction of an $n$-dimensional cube in Equation (3.10), the dependence of ${ }^{n} t_{c, 0.99}$ on $n$ is estimated from the inequality in Equation $(4.7 c)$. The numerically determined dependence of ${ }^{n} t_{c, 0.99}$ on $n$ in Equation (4.7a) was supported by the analytical estimates of the dependence of ${ }^{n} t_{c, 0.99} \mathrm{On} n$ in Equations (4.7b) and (4.7c).Therefore, ${ }^{n} t_{c, 0.99}$ was found to be proportional to $n$ to the power of 0.125 . 


\section{Nanomedicine \& Nanotechnology Open Access}

$$
\begin{aligned}
& { }_{t_{c, 0.99}}=n^{-1.25} \frac{20}{\pi^{2}} \frac{a^{2}}{\chi} \\
& n_{{ }_{c}, 0.99} \approx n^{-1.00} \frac{20}{\pi^{2}} \frac{a^{2}}{\chi} \\
& n^{-2.00} \frac{20}{\pi^{2}} \frac{a^{2}}{\chi}<{ }^{n}{ }_{t, 0.99}<n^{-1.00} \frac{20}{\pi^{2}} \frac{a^{2}}{\chi}, n>1
\end{aligned}
$$

The heat influx proportional to the temperature gradient at the surface $|\partial T / \partial r|_{r=a}$ begins at an infinitely large value and approaches zero after $t={ }^{n} t_{c, 0.99}$. The decrease in $|\partial T / \partial r|_{r=a}$ with time I for $n=1,10,50$, and 100 are compared in Figure 8(a), where the horizontal and vertical axes are the time normalized by ${ }^{n} t_{c, 0.99}$ and $\log _{10}|\partial T / \partial r|_{r=a}$, respectively. From the analytical solutions of Equation (3.2), $|\partial T / \partial r|_{r=a}$ is expected to decrease exponentially with the time for $1 \leq n \leq 3$. Numerical simulations for $4 \leq n \leq 100$ show that $|\partial T / \partial r|_{r=a}$ also decreases exponentially with the time. Although $|\partial T / \partial r|_{r=a}$ always decreases exponentially with the time, the decrease in $\Delta T_{\text {s-c }}$ is biased to the final stage for higher $n$ while $\Delta T_{\text {s-c }}$ decreases exponentially with time for $n \leq 3$, as shown in Figure 7(b). Because $|\partial T / \partial r|_{r=a}$ is higher at the initial stage, the measurement of $|\partial T / \partial r|_{r=a}$ in terms of $T_{\mathrm{s}} / a$ characterizes the influx period. Three influx periods ${ }^{n} t_{\mathrm{f}, 1.00},{ }^{n} t_{\mathrm{f}, 0.50}$, and ${ }^{n} t_{\mathrm{f}, 0.01}$ were defined as the times when $|\partial T / \partial r|_{r=a}$ reaches $1.0 T_{\mathrm{s}} / a, 0.5 T_{\mathrm{s}} / a$, and $0.01 T_{\mathrm{s}} / a$, respectively. ${ }^{n} t_{\mathrm{f}, 1.00},{ }^{n} t_{\mathrm{f}, 0.50}$, and ${ }^{n} t_{\mathrm{f}, 0.01}$ decrease with $n$, as shown in Figure 8(b), where the horizontal and vertical axes are $\log _{10}(n)$ and $\log _{10}\left({ }^{n} t_{f, 1.00}\right), \log _{10}\left({ }^{n} t_{f, 0.50}\right)$, and $\log _{10}\left({ }^{n} t_{t, 0.01}\right)$, respectively. The three influx periods for $1 \leq n \leq 3$ can be derived analytically in a similar manner as Equation (3.6b). Because there is a linear relation between $\log _{10}(n)$ and $\log _{10}\left({ }^{n} t_{\mathrm{f}, 0.01}\right)$ in Figure $8(\mathrm{~b}),{ }^{n} t_{\mathrm{f}, 0.01}$ is expected to be proportional to $n$ to the power index of ${ }^{n} \delta_{\mathrm{f}, 0.01}$. Because ${ }^{n} \delta_{\mathrm{f}, 0.01}$ was calculated from ${ }^{n} \mathrm{t}_{\mathrm{f}, 0.01}{ }^{n-1} t_{\mathrm{f}, 0.01}=n^{\delta} /(n-1)^{\delta}, \quad$ i.e., $\quad{ }^{n} \delta_{\mathrm{f}, 0.01}=\log _{10}\left({ }^{n} t_{\mathrm{f}, 0.01} /{ }^{n-}\right.$ $\left.{ }^{1} t_{\mathrm{f}, 0.01}\right) / \log _{10}\{n /(n-1)\},{ }^{n} \delta_{\mathrm{f}, 0.01}$ cannot be defined for one dimension. The dependence of ${ }^{n} \delta_{\mathrm{f}, 0.01}$ on $n$ is plotted in Figure 8(c), where the horizontal and vertical axes are $\log _{10}(n)$ and ${ }^{n} t_{f, 0.01}$, respectively. The rate of change in $\left|{ }^{n} \delta_{\mathrm{f}, 0.01}\right|$ exceeds $50 \%$ because ${ }^{2} \delta_{\mathrm{f}, 0.01}=-1.22$ at $n=2$ and ${ }^{100} \delta_{\mathrm{f}, 0.01}=-1.78$ at $n=100 \cdot{ }^{n} \delta_{\mathrm{f}, 0.01}$ was assumed to be -1.5 , which is the average of ${ }^{2} \delta_{\mathrm{f}, 0.01}$ and ${ }^{100} \delta_{\mathrm{f}, 0.01}$. Although the heat influx is higher at the initial stage, the stationary flat profile is almost completed after $t={ }^{n} \delta_{\mathrm{f}, 0.01}$ in a similar manner as the full profile completion after $t={ }^{n} \delta_{c, 0.99}$. Therefore, ${ }^{n} \delta_{\mathrm{f}, 0.01}$ was found to be expressed as a power law as follows:

$$
{ }^{n} t_{f, 0.01}=n^{-1.50} \frac{21.2}{\pi^{2}} \frac{a^{2}}{\chi}
$$

Where $21.2=4 \log _{\mathrm{e}}(200)$, which is derived from $|\partial T / \partial r|_{r=a}$ in the analytical solution of Equation(3.4a). Equation (4.8) is supported by Equation (4.7c) in a similar manner as ${ }^{n} t_{c, 0.99}$. Considering the finding that the rate of change in ${ }^{n} \delta_{\mathrm{f}, 0.01} \mid$ exceeds $50 \%$, it was concluded that the power law of ${ }^{n} t_{f, 0.01}$ in Equation (4.8) is inferior to that of ${ }^{n} t_{c, 0.99}$ in Equation (4.7a). There is an essential difference between Figure 7(b) and Figure 8(a); that is, the center filling and surface influx obey spherical and planar geometries, respectively. ${ }^{n} t_{c, 0.99}$ can be well-described by the concise power law of $n$ in Figure 7(d) and Equation (4.7c) owing to the effect of the spherical geometry at the center, whereas ${ }^{n} t_{c, 0.99}$ cannot be well-described by the concise power law of $n$ in Figure 8(c) and Equation (4.8) owing to the insufficient effect of the spherical geometry on the surface.

$T_{\mathrm{o}}$ increases exponentially with the time at the full profile completion period at $t={ }^{n} t_{\mathrm{c}, 0.99}$, as shown in Figure $7(\mathrm{~b}) .|\partial T / \partial r|_{r=a}$ almost always decreases exponentially with time, as shown in Figure 8(a). Thus, the coefficient $k_{\text {cent,fin }}$ representing the exponential increase in $T_{\mathrm{o}}$ and the coefficient $k_{\text {surffin }}$ representing the exponential decrease in $|\partial T / \partial r|_{r=a}$ are respectively defined in the difference scheme at $t={ }^{n} t_{\mathrm{c}, 0.99}$ as follows:

$$
\begin{aligned}
& T_{0}^{m} \cdot e^{+k_{c e n t}, \text { fin }} \Delta t=T_{0}^{m+1} \\
& \left(\frac{T_{k_{\max }}^{m}-T_{k_{\max -1}}^{m}}{\Delta r}\right) \cdot e^{-k_{\text {surf }, \text { fin }} t}=\left(\frac{\left.T_{k_{\max }^{m+1}-T_{k_{\max -1}}^{m+1}}^{\Delta r}\right)}{\Delta r}\right)
\end{aligned}
$$

Where $m+1$ and $m$ denote the final time step and the time step before the final time step, respectively. The dependencies of $k_{\text {cent,fin }}$ and $k_{\text {surf,fin }}$ on $n$ are plotted in Figure 8(d), where the horizontal and vertical axes are $n$ and the coefficients, respectively. Because there is almost a linear relation between $\log _{10}(n)$ and $\log _{10}\left(k_{\text {cent,fin }}\right)$ and $\log _{10}\left(k_{\text {surf,fin }}\right), k_{\text {cent,fin }}$ and $k_{\text {surf,fin }}$ can be described by power laws as

$$
\begin{aligned}
& k_{\text {cent }, \text { fin }}=2.46 \times 10^{-0} \cdot n^{1.57} \\
& k_{\text {surf ,fin }}=2.45 \times 10^{-2} \cdot n^{1.51}
\end{aligned}
$$




\section{Nanomedicine \& Nanotechnology Open Access}

The powers of 1.57 and 1.51 for $k_{\text {cent,fin }}$ and $k_{\text {surf,fin }}$ in Equation (4.10) are similar, where both $|\partial T / \partial r|_{r=a}$ and $T_{\mathrm{s}}-T_{\mathrm{o}}$ are close to zero at $t={ }^{n} t_{\mathrm{c}, 0.99}$. Although $|\partial T / \partial r|_{r=a}$ decreases exponentially with the time in a similar manner as planar thermal conduction, Equation (4.10) indicates that $k_{\text {cent,fin }}$ and $k_{\text {surf,fin }}$ change with $n$ with similar power indices. Therefore, it was concluded that the changes in the temperature profiles with the time at both the surface and center at $t={ }^{n} t_{c, 0.99}$ were determined in an equivalent manner by the effect of the spherical geometry indicated by $n-1$ despite the effect of the planar geometry on the surface.

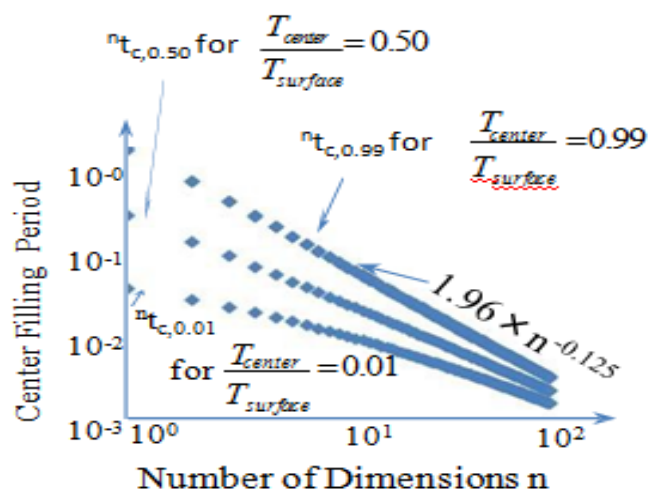

(a)

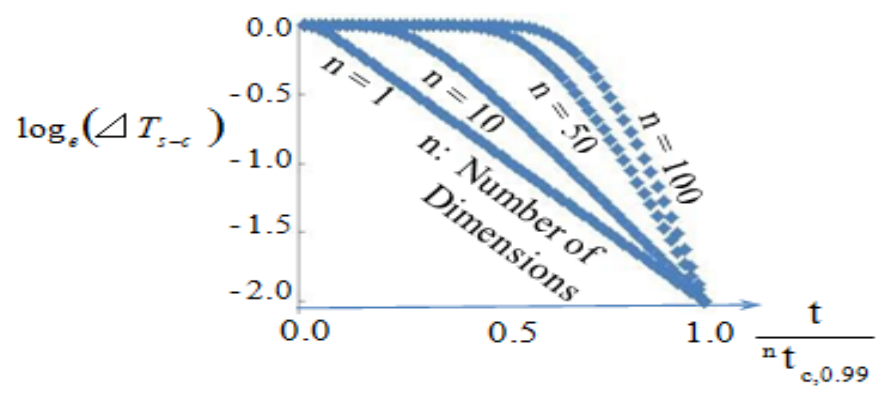

(b)

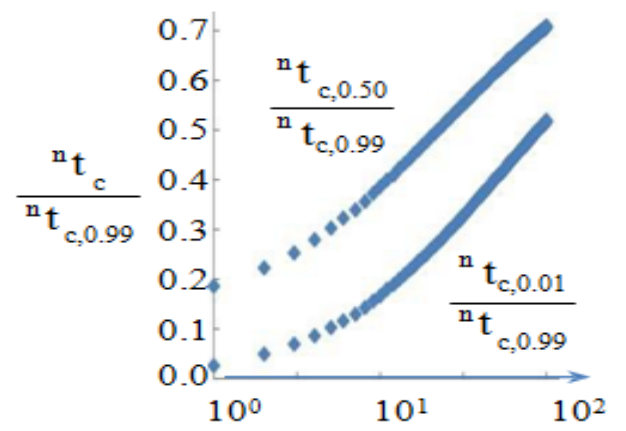

Number of Dimensions $\mathrm{n}$

(c)

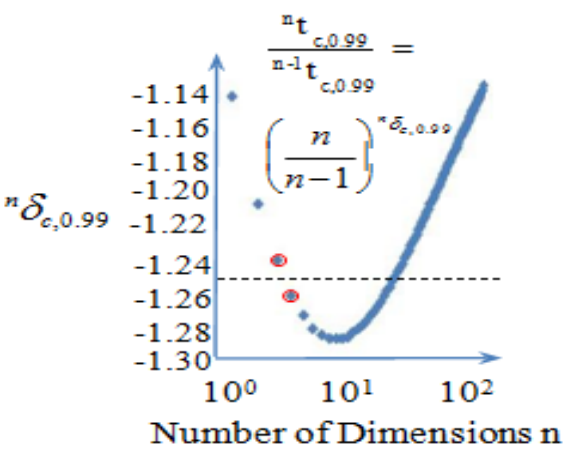

(d)

Figure 7: (a) Because the temperature is the lowest at the center, a center filling period exists at the final growth stage. The relationship between the number of dimensions $n$ and the three center filling periods ${ }^{n} t_{c, 0.99},{ }^{n} t_{c, 0.50}$, and ${ }^{n} t_{c, 0.01}$, defined as the times when the temperature $T_{\mathrm{o}}$ at the center of the sphere reaches $0.99 T_{\mathrm{s}}, 0.50 T_{\mathrm{s}}$, and $0.01 T_{\mathrm{s}}$, respectively, where $T_{0}=T_{r=0}$ and $T_{\mathrm{S}}=T_{r=a}$, are plotted. The final flat temperature profile is completed after the full profile completion time $t={ }^{n} t_{\mathrm{c}, 0.99}$. (b) The temperature difference between the surface and the center, defined as $\Delta T_{\mathrm{s}-\mathrm{c}}=\left(T_{\mathrm{s}}-T_{\mathrm{o}}\right) / T_{\mathrm{s}}$, decreases with time. When the time is normalized by ${ }^{n} t_{\mathrm{c}, 0.99}$, center filling was visually recognized to be biased to the final growth stage for $n \geq 9$, while that begins continuously from the initial growth stage for $n \leq 8$. In the analytical solutions for $n=1,2$, and 3, $\Delta T_{\mathrm{s}-\mathrm{c}}$ decreases exponentially with the time. (c) The degree of the bias of center filling to the final stage is evaluated by ratios ${ }^{n} t_{c, 0.50} /{ }^{n} t_{c, 0.99}$ and ${ }^{n} t_{c, 0.01} /{ }^{n} t_{c, 0.99}$, which approach asymptotic lines for $n \geq 9$. (d) The full profile completion period ${ }^{n} t_{c, 0.99}$ is proportional to $n$ to the power index of ${ }^{n} \delta_{c, 0.99}=$ $\log _{10}\left({ }^{n} t_{c, 0.99} /{ }^{n-1} t_{c, 0.99}\right) / \log _{10}\{n /(n-1)\}$. Because the rate of change in $\left|{ }^{n} \delta_{c, 0.99}\right|$ remains within $15 \%$ from $n=2$ to $n=100$, ${ }^{n} \delta_{c, 0.99}$ was assumed to be -1.25 , which is the average of ${ }^{4} \delta_{c, 0.99}$ and ${ }^{5} \delta_{c, 0.99}$. 


\section{Nanomedicine \& Nanotechnology Open Access}

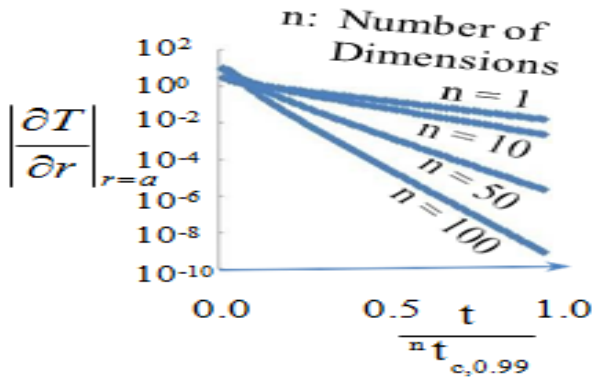

(a)

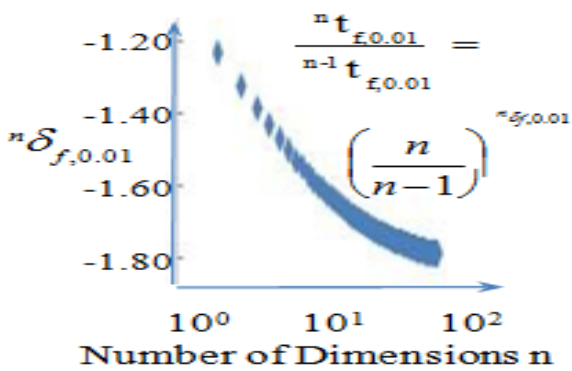

(c)

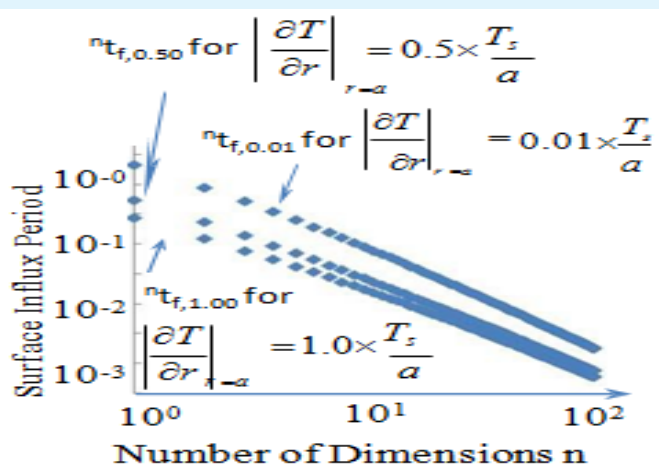

(b)

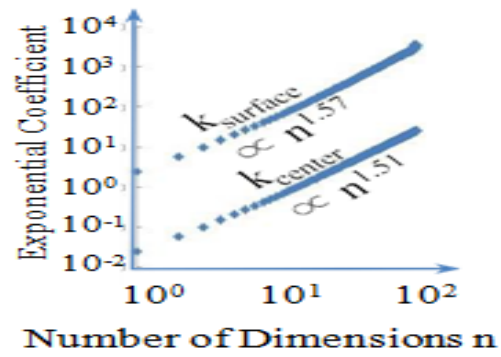

(d)

Figure 8: (a) The absolute value of the temperature gradient at the surface $|\partial T / \partial r|_{r=a}$ is $+\infty$ at $t=0$ and decreases exponentially with the time from $n=1$ to $n=100$, where the surface heat influx is proportional to $|\partial T / \partial r|_{r=a}$. (b) After the three influx periods of ${ }^{n} t_{\mathrm{f}, 0.01},{ }^{n} t_{\mathrm{f}, 0.50}$, and ${ }^{n} t_{\mathrm{f}, 1.00},|\partial T / \partial r|_{r=a}$ decreases from $\infty$ at $t=0$ to $0.01 T_{\mathrm{c}} / a, 0.50 T_{\mathrm{c}} / a$, and $1.00 T_{\mathrm{c}} / a$, respectively. The three influx periods decrease with $n$. The temperature profile is almost flat around the surface $(r=a)$ after $t \geq{ }^{n} t_{\mathrm{f}, 0.01}$. (c) The influx period ${ }^{n} t_{\mathrm{f}, 0.01}$ is proportional to $n$ to the power index of ${ }^{n} \delta_{\mathrm{f}, 0.01}$. (d) The coefficients of $k_{\text {surf,fin }}$ representing the exponential decrease in $|\partial T / \partial / r|_{r=a}$ with the time and $k_{\text {cent,fin }}$ representing the exponential increase in $T(r=0)$ with the time depend on $n$ as $n^{1.57}$ and $n^{1.51}$, respectively at $t={ }^{n} t_{c, 0.99}$.

\section{Motion of the Inflection Point and the Degree of Upward Convexity in the Profile}

The temperature profile for planar or slab heating exhibits a convex downward curve, as discussed in Sec. 2. Higher-dimensional spherical heating exhibits both convex upward and downward curves owing to the advection term in the thermal conduction equation in Equation (2.6). The inflection point $\partial^{2} T / \partial r^{2}=0$ between the convex upward and downward profiles is created at the surface of the sphere at $t=0$ and is then shifted toward the center after $t=0$. The changes in the radial position of the inflection point with the time for $n=1,2,3$, and 5 and $n=10,30$, and 100 are shown in Figures 9(a) and (b), respectively, where the horizontal and vertical axes are $t$ and $\log _{10}(r / a)$, respectively, and $r / a$ is the radial-position-to-radius ratio. Centripetal motion of the inflection point was found to occur intensively during the initial stage $\left(0 \leq t<{ }^{n} t_{c, 0.99} / 2\right)$ for $n \leq 7$ (Figure 9(a)) and continued to the final stage $\left({ }^{n} t_{c, 0.99} / 2 \leq t \leq{ }^{n} t_{c, 0.99}\right)$ for $n \geq 8$ (Figure 9(b)). The inflection point was found to reach the closest approaching distance ${ }^{n} r_{\text {inf,min }}$ from the center at $t={ }^{n} t_{c, 0.99}$. In the case of planar heating for $n=1$, ${ }^{1} r_{\text {inf,min }} / a=1$ because no inflection points are created (Figure 9(a)). The relation between $r_{\text {inf,min }} / a$ and $n$ is shown in Figure 9(c), where the horizontal and vertical axes are $\log _{10}(n)$ and $\log _{10}\left({ }^{n} r_{\mathrm{inf}, \min } / a\right)$, respectively. ${ }^{n} r_{\text {inf,min }} / a$ decreases from ${ }^{1} r_{\text {inf,min }} / a=1.0$ at $n=1$ to ${ }^{100} r_{\text {inf,min }} / a=0.2$ at $n=100$, and there is a linear relation between $\log _{10}(n)$ and $\log _{10}\left({ }^{n} r_{\text {inf,min }} / a\right)$. Thus, ${ }^{n} r_{\text {inf,min }} / a$ is 


\section{Nanomedicine \& Nanotechnology Open Access}

expected to be proportional to $n$ to the power index of ${ }^{n} \delta_{\text {inf,min. }} \quad{ }^{n} \delta_{\text {inf, min }}$ was calculated from ${ }^{n} \delta_{\text {inf,min }}=\log _{10}\left({ }^{n} r_{\text {inf,min }} /{ }^{n-1} r_{\text {inf,min }}\right) / \log _{10}\{n /(n-1)\} . \quad$ The dependence of ${ }^{n} \delta_{\text {inf,min }}$ on $n$ is plotted in Figure 8(d), where the horizontal and vertical axes are $\log _{10}(n)$ and ${ }^{n} \delta_{\text {inf,min, }}$ respectively. Although ${ }^{n} \delta_{\text {inf,min }}$ changes with $n$ from -0.382 to -0.355 for $2 \leq n \leq 9,{ }^{n} \delta_{\text {inf,min }}$ remains around -0.354 for $10 \leq n \leq 100$. Because the rate of change in $\left|{ }^{n} \delta_{\text {inf,min }}\right|$ remains within $9 \%$ from $n=2$ to $n=100$, a power law was determined, wherein ${ }^{n} \mathrm{r}_{\text {inf,min }} / a$ was proportional to $n$ to the power of ${ }^{n} \delta_{\text {inf,min }}=-0.354$. Therefore, $r_{\text {inf,min }} / a$ affected by the spherical geometry was found be described by the following power law:

$$
\frac{{ }^{n} r_{\text {inf,min }}}{a}=n^{-0.354}
$$

Because $0.5=7.08^{-0.354}$, the inflection point ${ }^{n} r_{\text {inf,min }}$ reaches within half of the radius $a$ from the center for $n \geq 8$. Thus, the degree of upward convexity is determined to be distinct for $n \geq 8$.

The advection term $(n-1)(1 / r)(\partial T / \partial r)$ in Equation (2.6) generates the convex upward part in the profile curve for $n \geq 2$, adding to the diffusion term $\left(\partial^{2} T / \partial r^{2}\right)$ that causes the convex downward profile. Although an inflection point is always generated for $n \geq 2$, the degree of upward convexity is distinct for $n \geq 9$, as shown in Figures 4(a6)-(a8), and not distinct for $2 \leq n \leq 5$, as shown in Figures 4(a2)-(a5). The degree of upward convexity is compared with profiles for $n=2,4,8,9,30$, and 100 at the times when $t={ }^{n} t_{\mathrm{f}, 1.00}\left(|\partial T / \partial r|_{r=a}=1.0 T_{\mathrm{s}} / a\right)$, $t={ }^{n} t_{\mathrm{t}, 0.50}\left(|\partial T / \partial r|_{r=a}=0.5 T_{\mathrm{s}} / a\right)$, and $t={ }^{n} t_{\mathrm{f}, 0.01}\left(|\partial T / \partial r|_{r=a}=0.01 T_{\mathrm{s}} / a\right)$ in Figures 4(b1), 4(b2), and 4(b3), respectively, where the horizontal and vertical axes are the radial position from $r=a$ to $r=0$ and $T$, respectively. The temperature $T_{\mathrm{o}}{ }^{\prime}$ at the center is defined as $T_{\mathrm{o}}{ }^{\prime}=T_{\mathrm{s}}-|\partial T / \partial r|_{r=a} a$; that is, the temperature decreases from the surface while maintaining the same temperature gradient at the surface $|\partial T / \partial r|_{r=a}$ toward the center. Temperature profiles with the same surface gradient $|\partial T / \partial r|_{r=a}$ are compared at the three times in Figure 4(b), where straight dotted lines connecting $T_{\mathrm{s}}(r=a)$ and $T_{\mathrm{o}}{ }^{\prime}(r=0)$ are plotted at the three times. The profile curve deviating from the dotted line and then approaching the bottom $(T=0)$ as $r$ decreases can be evaluated by a curve of the degree of upward convexity. Because the temperature profile always has a convex downward part round the center, the temperature profile exhibits a convex upward part around the surface for $n \geq 2$.

$$
\frac{n_{t_{f, 1.00}}}{n_{t_{c, 0.01}}}=4.44 \times n^{-0.708}
$$

The dependencies of ${ }^{n} t_{\mathrm{f}, 1.00} /{ }^{n} t_{\mathrm{c}, 0.01}$ and ${ }^{n} t_{\mathrm{f}, 0.01} /{ }^{n} t_{\mathrm{c}, 0.99}$ on $n$ are plotted in Figure 10(a), where the horizontal and vertical axes are $\log _{10}(n)$ and $\log _{10}\left({ }^{n} t_{f, 1.00} /{ }^{n} t_{c, 0.01}\right)$ and $\log _{10}\left({ }^{n} t_{\mathrm{f}, 0.01} /{ }^{n} t_{c, 0.99}\right)$, respectively. The degree of upward convexity in the temperature profile is high when ${ }^{n} t_{\mathrm{f}, 0.01} /{ }^{n} t_{c, 0.99}<1$; that is, the surface temperature gradient is almost flat $\left(|\partial T / \partial r|_{r=a}=0.01\right)$ at $t={ }^{n} t_{\mathrm{f}, 0.01}$ at first, and, a long time afterward, the center temperature profile is almost flat $\left(T_{0}=0.99 T_{\mathrm{s}}\right)$ at $t={ }^{n} t_{c, 0.99}$. When ${ }^{n} t_{\mathrm{f}, 1.00} /{ }^{n} t_{\mathrm{c}, 0.01} \geq 1$ for a lower $n, T_{\mathrm{o}}$ reaches $0.01 T_{\mathrm{s}}$ before $|\partial T / \partial r|_{r=a}$ reaches $1.00 T_{s} / a$. The degree of upward convexity in the temperature profile is high when ${ }^{n} t_{\mathrm{f}, 1.00} /{ }^{n} t_{\mathrm{c}, 0.01}<<1$; that is, the surface temperature gradient $|\partial T / \partial r|_{r=a}$ reaches $1.00 T_{\mathrm{c}} / a$ from $\infty$ at $t=0$ first, and, a long time afterward, the center temperature $T_{0}(r=0)$ reaches $0.01 T_{\mathrm{s}}(r=a)$. The decreases in ${ }^{n} t_{f, 1.00} /{ }^{n} t_{c, 0.01}$ and ${ }^{n} t_{f, 0.01} /{ }^{n} t_{c, 0.99}$ with the increase in $n$ indicate that the degree of upward convexity in the profile becomes higher with the increase in $n$. Thus, a profile with a lower degree of upward convexity can be discriminated from ${ }^{n} t_{\mathrm{f}, 1.00} /{ }^{n} t_{\mathrm{c}, 0.01} \geq 1$ and ${ }^{n} t_{\mathrm{f}, 0.01} /{ }^{n} t_{c, 0.99} \geq 1$. When ${ }^{n} t_{\mathrm{f}, 1.00} /{ }^{n} t_{c, 0.01}<1$ for $n \geq 9, T_{\mathrm{o}}$ reaches $0.01 T_{\mathrm{s}}$ after a certain time from the time when $|\partial T / \partial r|_{r=a}$ reaches $1.00 T_{s} / a$ on account of the convex upward profile. Thus, ${ }^{n} t_{\mathrm{f}, 1.00} /{ }^{n} t_{c, 0.01}$ and ${ }^{n} t_{f, 0.01} /{ }^{n} t_{c, 0.99}$ are expected to determine the degree of upward convexity in the profile. The decreases in ${ }^{n} t_{t, 1.00} /{ }^{n} t_{c, 0.01}$ and ${ }^{n} t_{t, 0.01} /{ }^{n} t_{c, 0.99}$ with the increase in $n$ indicate that the degree of upward convexity increases with the increase in $n$.

Because there is a linear relation between $\log _{10}(n)$ and $\log _{10}\left({ }^{n} t_{\mathrm{f}, 1.00} /{ }^{n} t_{c, 0.01}\right)$ in Figure 10(a), ${ }^{n} t_{\mathrm{f}, 1.00} /{ }^{n} t_{\mathrm{c}, 0.01}$ is expected to be proportional to $n$ to the power index of ${ }^{n} \delta_{\mathrm{fc}}$. Setting ${ }^{n} S_{\mathrm{fc}}={ }^{n} t_{\mathrm{f}, 1.00} /{ }^{n} t_{\mathrm{c}, 0.01}$, the power index ${ }^{n} \delta_{\mathrm{fc}}$ was calculated from ${ }^{n} S_{\mathrm{fc}} /{ }^{n-1} S_{\mathrm{fc}}=n^{\delta} /(n-1)^{\delta}$. The dependence of ${ }^{n} \delta_{\mathrm{fc}}$ on $n$ is plotted in Figure 10(b), where the horizontal and vertical axes are $\log _{10}(n)$ and ${ }^{n} \delta_{\mathrm{fc}}$, respectively. ${ }^{n} \delta_{\mathrm{fc}}$ ranges from -0.734 at $n=2$ and 100 to -0.681 at $n=5$. Because the rate of change in $\left|{ }^{n} \delta_{\mathrm{fc}}\right|$ remains within $8 \%$ from $n=2$ to $n=100$, a power law was determined, wherein ${ }^{n} t_{f, 1.00} /{ }^{n} t_{c, 0.01}$ was proportional to $n$ to the power of -0.708 , which is the average of -0.734 and -0.681 . Because Equation (3.4a) and Equation (3.5) indicate that ${ }^{1} t_{\mathrm{f}, 1.00}=2.828 \times 10^{-1}$ and ${ }^{1} t_{\mathrm{c}, 0.01}=6.370 \times 10^{-2}$, respectively, ${ }^{1} t_{\mathrm{t}, 1.00} /{ }^{1} t_{\mathrm{c}, 0.01}=4.44$. Thus, ${ }^{n} t_{\mathrm{f}, 1.00} /{ }^{n} t_{\mathrm{c}, 0.01}$ was described by the following power law: 


\section{Nanomedicine \& Nanotechnology Open Access}

Because ${ }^{n} t_{f, 1.00} /{ }^{n} t_{c, 0.01}=1.0$ at $n=8.21$ in Equation (4.12), the degree of upward convexity in the profile was determined to be distinct for $n \geq 9$. Because ${ }^{n} t_{\mathrm{f}, 0.01} /{ }^{n} t_{\mathrm{c}, 0.99}$ deviates distinctly from 1.0 for $n \geq 9$ while ${ }^{n} t_{\mathrm{f}, 0.01}{ }^{n} t_{\mathrm{c}, 0.99}$ is around 1.0 for $n \leq 8$ in Figure $10(\mathrm{a}),{ }^{n} t_{\mathrm{f}, 0.01} /{ }^{n} t_{\mathrm{c}, 0.99}$ also indicates the degree of upward convexity in the profile. Because ${ }^{n} t_{f, 1.00} /{ }^{n} t_{c, 0.01}$ is expressed by the concise power law of $n$ as Equation (4.12), ${ }^{n} t_{f, 1.00} /{ }^{n} t_{c, 0.01}$ can indicate the degree of upward convexity in the profile more effectively than ${ }^{n} t_{f, 0.01} /{ }^{n} t_{c, 0.99}$. Therefore, the degree of upward convexity was determined numerically by ${ }^{n} t_{f, 1.00} /{ }^{n} t_{c, 0.01}$.

Figure 8(a) shows that the surface temperature gradient $|\partial T / \partial r|_{r=a}$ decreases exponentially with the time. The dependence of the final gradient $|\partial T / \partial r|_{r=a}$ at $t={ }^{n} t_{c, 0.99}$ on $n$ is shown in Figure 10(c), where the final gradient $|\partial T / \partial r|_{r=a}$ almost remains similar around $10^{-2}$ for $n \leq 8$ and decreases distinctly for $n \geq 9$. This agrees with three facts: Figure 7 indicating that the center filling is biased to the final stage for $n \geq 9$, Figure 9 indicating that the inflection point finally reaches within half of the radius for $n \geq 8$, and Figure 10 indicating that the surface gradient becomes flat much faster than the center temperature profile becomes flat for $n \geq 9$. Therefore, the almost similar convex downward temperature profile and the heat flux per unit surface $|\partial T / \partial r|_{r=a}$ approach the final flat profile for $t=0$ to ${ }^{n} t_{c, 0.99}$ for $1 \leq n \leq 8$; that is, the change in the profile and the heat transfer are similar with the time scale of ${ }^{n} t_{c, 0.99}$ for $1 \leq n \leq 8$. Although there are no analytical solutions for $n>3$, numerical simulations of the thermal conduction in four- and fivedimensional spheres, which are related to the fluid rotation in the next section, are supported by the analytical solution well for $1 \leq n \leq 3$ using the similarity about $n$.

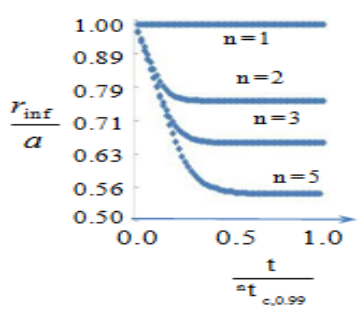

(a)

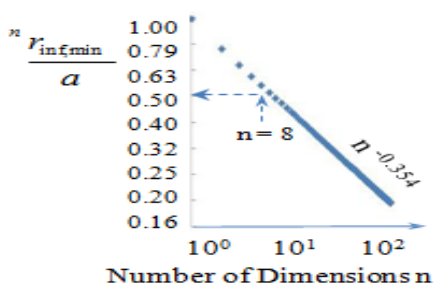

(c)

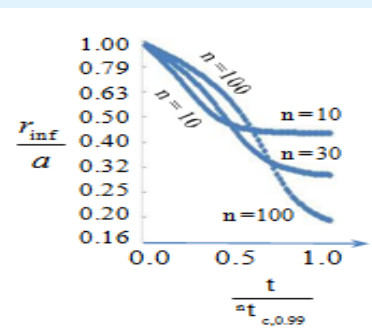

(b)

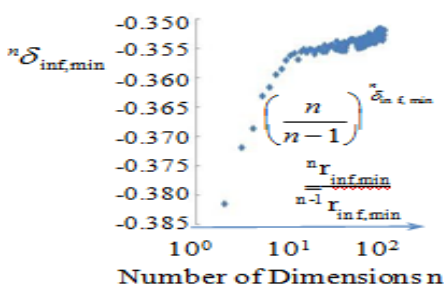

(d)

Figure 9: The inflection point $\partial^{2} T / \partial r^{2}=0$ between the convex upward and downward profile curves moves from the surface toward the center. The change in the radial position $r_{\text {inf }}$ of the inflection point with the time is shown for (a) $n=1,2,3$, and 5 and (b) $n=10,30$, and 100. The inflection point moves intensively in the initial stage $\left(0 \leq t<{ }^{n} t_{c, 0.99} / 2\right)$ only or moves continuously from the initial stage to the final stage $\left({ }^{n} t_{c, 0.99} / 2 \leq t \leq{ }^{n} t_{c, 0.99}\right)$ for $n \leq 7$ or $n \geq 8$, respectively. (c) At the full profile completion period $t={ }^{n} t_{c, 0.99}$, the inflection point reaches the closest approaching distance $r_{\mathrm{inf}, \min }$ from the center, which decreases with $n$ and is less than half of the radius for $n \geq 8$. (d) The closest distance $r_{\text {inf,min }} / a$ is proportional ton to the power index of ${ }^{n} \delta_{\text {inf,min. }}$. Because the rate of change in $\left|{ }^{n} \delta_{\text {inf,min }}\right|$ remains within $9 \%$ from $n=2$ to $n=100$ and within $1 \%$ from $n=10$ to $n=100,{ }^{n} \delta_{\text {inf,min }}$ was assumed to be -0.354 . 


\section{Nanomedicine \& Nanotechnology Open Access}

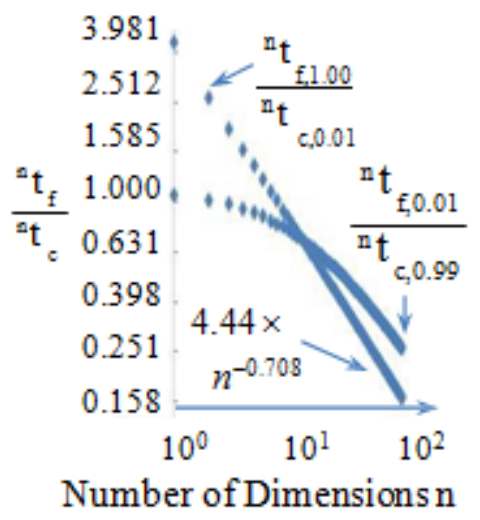

(a)

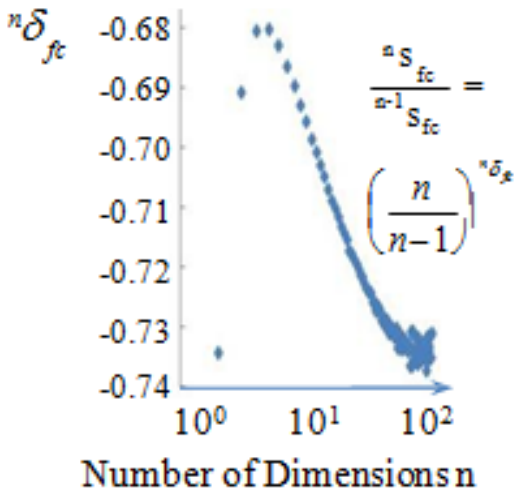

(b)

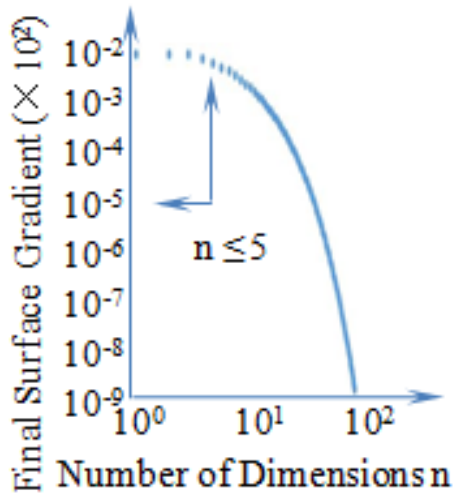

(c)

Figure 10: (a) The decreases in ${ }^{n} t_{\mathrm{f}, 1.00} /{ }^{n} t_{\mathrm{c}, 0.01}$ and ${ }^{n} t_{\mathrm{f}, 0.01} /{ }^{n} t_{\mathrm{c}, 0.99}$ with the increase in $n$ indicate that the degree of upward convexity in the profile becomes higher with the increase in $n$. The degree of upward convexity in the temperature profile is high when ${ }^{n} t_{\mathrm{f}, 0.01} /{ }^{n} t_{c, 0.99}<<1$; that is, the surface temperature gradient is almost flat $\left(|\partial T / \partial r|_{r=a}=0.01\right)$ at $t={ }^{n} t_{\mathrm{f}, 0.01}$ at first, and, a long time after that, the center temperature profile is almost flat $\left(T_{\mathrm{o}}=0.99 T_{\mathrm{s}}\right)$ at $t={ }^{n} t_{\mathrm{c}, 0.99}$. (b) The ratio ${ }^{n} t_{\mathrm{f}, 1.00} /{ }^{n} t_{\mathrm{c}, 0.01}$ is proportional to $n$ to the power index of ${ }^{n} \delta_{\mathrm{fc}}$. Because the rate of change in $\left|{ }^{n} \delta_{\mathrm{fc}}\right|$ remains within $8 \%$ from $n=2$ to $n=100,{ }^{n} t_{\mathrm{f}, 1.00} /{ }^{n} t_{\mathrm{c}, 0.01}$ could be expressed as ${ }^{n} t_{\mathrm{f}, 1.00} /{ }^{n} t_{\mathrm{c}, 0.01}=4.44 n^{-0.708}$. The degree of upward convexity in the temperature profile, which was visually recognized in Figure 4 , can be numerically evaluated to be higher for ${ }^{n} t_{\mathrm{f}, 1.00} /{ }^{n} t_{\mathrm{c}, 0.01}<1$ and $n \geq 9$ and lower for ${ }^{n} t_{\mathrm{f}, 1.00} /{ }^{n} t_{\mathrm{c}, 0.01}>1$ and $n \leq 8$. (c) Because the final surface temperature gradient $|\partial T / \partial r|_{r=a}$ at $t={ }^{n} t_{c, 0.99}$ is over half of the gradient of slab heating for $n \leq 6$, the heat transfer is almost similar with respect to the magnitude of ${ }^{n} t_{c, 0.99}$ for $n \leq 6$. The heat transfer changes drastically for $n \geq 7$.

\section{Fluid Rotations Surrounded by the Surfaces of a Disc, Cylinder, and Sphere}

A cylinder with diameter $2 a$ is immersed in a Newtonian fluid with a density $\rho$ and viscosity $\mu$, and the coordinate system is shown in Figure 3(e). When the cylinder starts to move in the $z$ direction with a constant velocity $U$ at $t=0$, cylindrical laminar flow layers move with the axial velocity $u_{z}(r)$ and diffuse to the central axis from the cylinder; these layers finally approach a flat flow profile with $U$. The growth of the flow is analogous to cylindrical (two-dimensional spherical) heating from the surface. The exchange of $T$ with $u_{z}(r)$ and $\chi$ with $v(=\mu / \rho)$ in Equation $(3.2 b)$ yields the growth of the flow shown in Figure 4(a2). The torque equations for fluid rotations for the disc, cylinder, and sphere were found to be equivalent to the thermal conduction of one-, four-, and fivedimensional spheres, respectively, as discussed in Sec. 2 .

Volumes with a radius of $a$ surrounded by two coaxial parallel discs, a cylinder, and a sphere with a radius of $a$ are filled with liquid water at $25^{\circ} \mathrm{C}$. When the two parallel discs, cylinder, and sphere start rotating with a constant angular velocity $\Omega$ (surface angular velocity) about their central axes at $t=0, \Omega$ changes into the angular velocity $\omega$ of the thin fluid layers within. Fluid rotation is induced by shaking the containers by hand, which is terminated at $t=0$. The attenuation of the fluid rotation after $t=0$ is visualized by light-irradiated suspensions. The duration of the attenuation of rotation is visually observed ten times after $t=0$. The shaking before $t=0$ was continued for twice the duration of attenuation observed after $t=0$. The growth periods ${ }^{1} t_{c, 0.01},{ }^{4} t_{c, 0.01}$, and ${ }^{5} t_{c, 0.01}$ were defined as the times when $\omega$ at the center increased to $0.01 \Omega$, which is the time when the velocity at the center is 0.01 of the surface angular velocity. The growth periods ${ }^{1} t_{c, 0.99}$, ${ }^{4} t_{c, 0.99}$, and ${ }^{5} t_{c, 0.99}$ were defined as the times when $\omega$ at the center reached $0.99 \Omega$, which is the full profile completion period. The growth periods ${ }^{n} t_{c, 0.01}$ and ${ }^{n} t_{c, 0.99}$ from $n=1$ to $n=5$ are normalized to $a^{2} / \chi$ in Table 1 . Because the fluid rotation induced by shaking in an arc is not actual rotation of the outer shell, the final state just after shaking is not fluid rotation with a uniform angular velocity $\Omega$. Thus, the duration of attenuation of the rotation was expected to an intermediate value between ${ }^{n} t_{c, 0.01}$ and ${ }^{n} t_{c, 0.99}$ by setting $v=0.897 \times 10^{-6}$ for water at $25^{\circ} \mathrm{C}$. Although 


\section{Nanomedicine \& Nanotechnology Open Access}

the Reynolds number was calculated to be around 1 [19], no vortexes besides the coaxial rotation were confirmed. Thus, the duration of attenuation can be estimated using ${ }^{n} t_{c, 0.01}$ and ${ }^{n} t_{c, 0.99}$ because of the laminar flow assumption, where the duration of attenuation does not to depend on the angular velocity (flow velocity).

Three spherical flasks (Hario, Japan) having spherical part volumes and radii of 50,100 , and $200 \mathrm{~mL}$, and 22.9 , 28.8 , and $36.3 \mathrm{~mm}$, respectively, were filled with water. Because the fluid rotation can be examined by analogy with the thermal conduction of a five-dimensional sphere, the two durations of ${ }^{5} t_{c, 0.01}$ and ${ }^{5} t_{c, 0.99}$ are the lower and upper parabolic curves, respectively, in Figure 11(a), where the horizontal and vertical axes are the radius of the sphere ranging from $a=0$ to $a=40 \mathrm{~mm}$ and the duration in seconds, respectively. The observed durations are plotted as dots in Figure 11(a). Because the observed durations were bounded by the upper ${ }^{5} t_{c, 0.99}$ and lower ${ }^{5} t_{c, 0.01}$ parabolic curves and proportional to the square of the radii of the spheres in a similar manner as the formulae for ${ }^{5} t_{c, 0.99}$ and ${ }^{5} t_{c, 0.01}$, the duration of the fluid rotation in the sphere was evaluated by the intermediate value of the two durations of ${ }^{n} t_{c, 0.01}$ and ${ }^{n} t_{c, 0.99}$ from thermal conduction of a five-dimensional sphere.

Two graduated cylinders (Shibata, Japan) with volumes and inner diameters $2 a$ of 50 and $200 \mathrm{~mL}$ and $2 a=22$ and $39 \mathrm{~mm}$ were filled with water to a depth of $h$. When the depth is lower than the diameter of the cylinder $(h \leq 2 a)$, the fluid is regarded as a fluid disc, and the fluid rotation at the bottom of the graduated cylinder can be examined by analogy with fluid disc rotations bounded by two parallel coaxial discs, which is one-dimensional spherical thermal conduction. The two durations of ${ }^{1} t_{\mathrm{c}, 0.01}$ and ${ }^{1} t_{\mathrm{c}, 0.99}$ are the lower and upper parabolic curves, respectively, in Figures $11(\mathrm{~b})$ and (c), where the horizontal and vertical axes are $h$ ranging from $h=0$ to $h=60 \mathrm{~mm}$ and the duration of rotation in seconds, respectively. Because the rotation of the inner part of the volume continues even after the surface layer terminates rotation, the existence of a surface force for terminating the surface rotation is expected. Therefore, it is considered that the surface of the water is not a force-free boundary and partially similar to the rigid boundary at the bottom. Thus, ${ }^{1} t_{c, 0.01}$ and ${ }^{1} t_{c, 0.99}$ in Figures 11(b) and (c) were calculated by assuming $a=h / 2$ in Table 1; that is, both the bottom and the surface of water are similar boundary discs. The observed durations are plotted with dots in Figures 11(b) and (c), where the left parts correspond to cases when $h \leq a$. The observed durations were bounded by the upper ${ }^{1} t_{c, 0.99}$ and lower ${ }^{1} t_{c, 0.01}$ parabolic curves. Therefore, the duration of the fluid rotation in the cylinder is considered to be evaluated from the thermal conduction of a onedimensional sphere (slab heating) if the depth is less than the diameter. If the surface of the water in the graduated cylinder is a force-free boundary, ${ }^{1} t_{c, 0.01}$ and ${ }^{1} t_{c, 0.99}$ should be calculated by assuming $a=h$ in Table 1 ; that is, ${ }^{1} t_{c, 0.01}$ and ${ }^{1} t_{c, 0.99}$ calculated with $a=h$ are four times those calculated with $a=h / 2$. Because the observed durations are distributed between the upper ${ }^{1} t_{c, 0.99}$ and lower ${ }^{1} t_{c, 0.01}$ parabolic curves calculated with $a=h / 2$, the surface of the liquid water is considered not to be a force-free boundary. When the depth is greater than the diameter of the cylinder $(h \geq 2 a)$, the fluid is regarded as a fluid cylinder. Because the fluid rotation can be examined by analogy with the thermal conduction of a fourdimensional sphere, the two durations of ${ }^{4} t_{c, 0.01}$ and ${ }^{4} t_{c, 0.99}$ are the lower and upper straight lines, respectively, in Figures 11(b) and (c). The observed durations were bounded by the upper ${ }^{4} t_{c, 0.99}$ and lower ${ }^{4} t_{c, 0.01}$ straight lines. Therefore, the duration of the fluid rotation in the cylinder is considered to be evaluated from the thermal conduction of a four-dimensional sphere if the depth is greater than the diameter.

The diameter and depth in one example of a cylindrical water container used for measuring the magnetic field uniformity in an MRI head coil are 180 and $250 \mathrm{~mm}$, respectively [4]. The duration of attenuation for rotation in a $\varnothing 39 \mathrm{~mm}$ cylinder with a depth of $55 \mathrm{~mm}$ shown in Figure $11(\mathrm{c})$ is about $80 \mathrm{~s}$, where the ratio $h: 2 a$ is similar to that of the water container, and $h \geq 2 a$ is satisfied. Because these sizes are $0.22(=39 / 180)$ of the water tank, the duration of attenuation of the water tank is expected to be $28 \mathrm{~min}$, which is calculated by dividing $80 \mathrm{~s}$ by $(0.22)^{2}$. Because the expected time for the complete termination of water rotation is about half an hour when using a cylindrical water container, a rectangular water container is recommended for measuring the field uniformity. 


\section{Nanomedicine \& Nanotechnology Open Access}

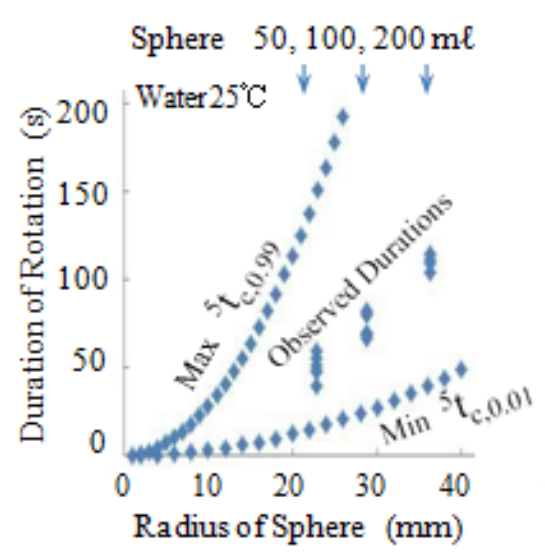

(a)

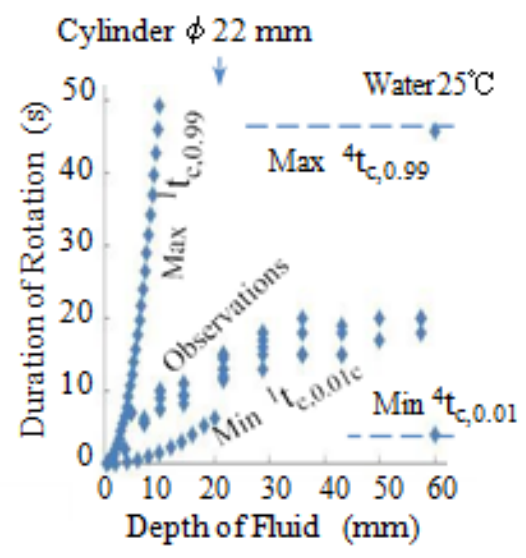

(b)

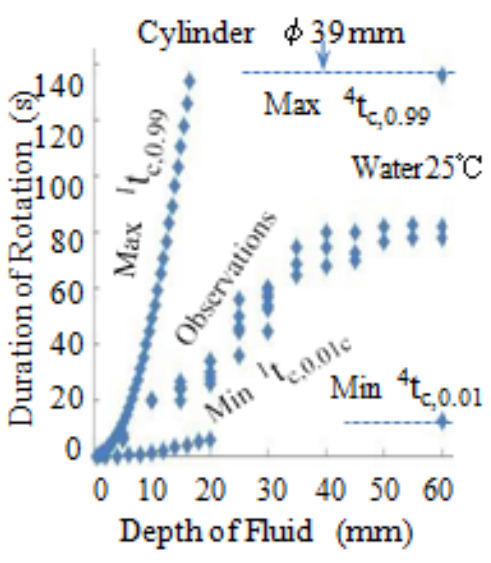

(c)

Figure 11: (a) The observed durations of fluid rotation in spherical flasks with volumes of 50, 100, and $200 \mathrm{~mL}$ are plotted as dots. The maximally and minimally expected durations of ${ }^{5} t_{c, 0.99}$ and ${ }^{5} t_{c, 0.01}$, which are proportional to $r^{2}$, are plotted as the upper and lower parabolic curves, respectively. (b), (c) The observed durations of fluid rotation in graduated cylinders with volumes of (b) 50 and (c) $200 \mathrm{~mL}$ are plotted as dots. For the case where the depth is less than the diameter of the cylinder $(h \leq 2 a)$, the maximally and minimally expected durations of ${ }^{1} t_{c, 0.99}$ and ${ }^{1} t_{c, 0.01}$, which are proportional to $h^{2}$, are plotted as the upper and lower parabolic curves, respectively. For the case where the depth is greater than the diameter of the cylinder $(h>2 a)$, the maximally and minimally expected durations of ${ }^{4} t_{c, 0.99}$ and ${ }^{4} t_{c, 0.01}$ are plotted as the upper and lower straight lines, respectively. The observed durations are bounded by the two parabolic curves and two straight lines.

\begin{tabular}{|c|c|c|}
\hline $\boldsymbol{n}$ & $\left({ }^{n} \mathbf{t}_{\mathbf{c}, 0.01}\right) /\left(\boldsymbol{a}^{2} / \chi\right)$ & $\left({ }^{n} \mathbf{t}_{\mathbf{c}, 0.99}\right) /\left(\boldsymbol{a}^{2} / \chi\right)$ \\
\hline 1 & $6.37 \times 10^{-2}$ & $1.97 \times 10^{-0}$ \\
\hline 2 & $4.78 \times 10^{-2}$ & $8.83 \times 10^{-1}$ \\
\hline 3 & $3.97 \times 10^{-2}$ & $5.40 \times 10^{-1}$ \\
\hline 4 & $3.43 \times 10^{-2}$ & $3.78 \times 10^{-1}$ \\
\hline 5 & $3.05 \times 10^{-2}$ & $2.85 \times 10^{-1}$ \\
\hline
\end{tabular}

Table 1: The dependencies of the growth periods ${ }^{n} t_{c, 0.01}$ and ${ }^{n} t_{c, 0.99}$ on the number of dimensions $n$ normalized to $a^{2} / \chi$, where $a$ and $\chi$ are the radius and thermal diffusivity, respectively.

\section{Conclusions}

Three water containers with the geometries of a disk, which is a cylinder whose height is sufficiently smaller than the bottom diameter; a cylinder whose bottom diameter is sufficiently smaller than its height; and a sphere filled with a Newtonian fluid are considered. When the three containers rotate, the laminar fluid layers, which are initially static, begin to coaxially rotate. The increases in the angular velocity of the laminar fluid layers in the three containers were found to be mathematically equivalent to the thermal conduction of one-, four-, and five-dimensional spheres heated from their surfaces, respectively, where the temperature is replaced with angular velocity. The thermal conduction of one-, two-, and three-dimensional spheres, heated from their surfaces was analytically obtained, where slab, cylindrical, and spherical thermal conduction were regarded as the heating modes of one-, two-, and three-dimensional spheres, respectively. The centrosymmetric thermal conduction equation of $n$-dimensional spheres has both advection $[\partial T / \partial t=\chi(n-1)(1 / r)(\partial T / \partial r)]$ and diffusion $\left[\partial T / \partial t=\chi \partial^{2} T / \partial r^{2}\right]$ terms, whereas that of the slab volume $(n=1)$ has only the diffusion $\left(\partial T / \partial t=\partial^{2} T / \partial r^{2}\right)$ term. A 


\section{Nanomedicine \& Nanotechnology Open Access}

step-like temperature profile is initially formed at the surface. Because the advection term shifts the step-like temperature profile with the centripetal velocity $(n-1)(1 / r)$ towards the center and the diffusion term always requires a convex downward profile in the entire region, the inward thermal conduction for $n \geq 2$ creates convex upward profile curves originating from the surface and moving toward the convex downward profile around the center. The full profile completion period ${ }^{n} t_{c, 0.99}$ required to achieve a final flat temperature profile, was defined as the time when the center temperature reaches $99 / 100$ of the surface temperature.

The completion periods numerically determined for $4 \leq n \leq 100$ and analytically determined for $1 \leq n \leq 3$ were found to be proportional to $n$ to the power of -1.25 $\left({ }^{n} t_{c, 0.99} 1 /{ }^{1} t_{c, 0.99}=n^{-1.25}\right)$, which is mainly determined for $4 \leq n \leq 5$ and within $9 \%$ accuracy for $1 \leq n \leq 100$. The power of -1.25 was approximately supported by the finding that the surface area-to-volume ratio was proportional to $n$ and analytically supported by the thermal conduction of $n$-dimensional cubes inscribed within and circumscribing an $n$-dimensional sphere in more detail. The numerical accuracy was checked by the residual evaluated from the difference between the total heat influx quantity at the surface and the total heat increase in the $n$-dimensional sphere after the full profile completion period. An adequate time step $\Delta t$ and spatial division $\Delta r$ were determined for numerical simulations for $4 \leq n \leq 100$ within an accuracy of $10^{-3}$. The stable simulation condition investigated by a von Neumann stability analysis for the diffusion $\left(\partial T / \partial t=\partial^{2} T / \partial r^{2}\right)$ term requires $\Delta t<(\Delta r)^{2} /(2 \chi)$ throughout the entire volume. The heat transport velocity $\chi(n-1)(1 / r)$ appearing in the advection term should be less than the numerical grid velocity $\Delta r / \Delta t$ because the CFL condition predicts unstable simulation results when $\chi(n-1)(1 / r)>\Delta r / \Delta t$. Thus, $\Delta t$ should be less than $r \Delta r /(n-1)$, and there is a possibility that $\chi(n-1)(1 / r)>\Delta r / \Delta t$ around $r=0$ (the center) at greater $n$, even though $\chi(n-1)(1 / r) \leq \Delta r / \Delta t$ is satisfied, except at the center. Nevertheless, a stable convergent simulation within an accuracy of $10^{-2}$ could be performed because the volume around the center, where the CFL condition does not hold, is significantly lower compared to the entire volume. It was found that the stable condition from the von Neumann stability analysis should hold in the entire volume, even though the CFL condition does not hold only near the center.

The thermal conduction initially and finally increased the temperature to the surface temperature $T_{\mathrm{s}}$ around the surface and center, respectively. The surface temperature gradient $|\partial T / \partial r|_{r=a}$, which is proportional to the surface heat transfer, decreased exponentially with the time, and the rate of decrease is almost similar for $1 \leq n \leq 5$. This means that the change in the temperature profile with the time is similar with respect to the full profile completion period ${ }^{n} t_{c, 0.99}$ for $1 \leq n \leq 5$ because the temperature profile is almost convex downward and similar to slab heating for the completion period. This certifies a sufficient accurate expectation of the numerical simulation of spherical thermal conduction for $4 \leq n \leq 5$, which is required for the fluid rotation analysis from the analytical solutions for $1 \leq n \leq 3$. Numerical simulation for $4 \leq n \leq 100$ revealed concise power laws of $n$ regarding the change in the temperature profile. These laws supported the numerical accuracy and enhanced the validity of the numerical simulations of four- and fivedimensional spheres, which will be applied to fluid rotation. The flow field around the rotating sphere or rod in a fluid, which is necessary for the precise evaluation of rotational Brownian motion, will be numerically simulated with accuracy on the basis of the thermal conduction of four- and five-dimensional spheres.

The degree of upward convexity in the development of the temperature profile during heating was visually recognized to be distinct for $6 \leq n$. The convex upward profile is related to the fact that the decrease in the temperature difference between the surface and the center is biased to the final stage for $6 \leq n$ while the temperature difference decreases exponentially with the time for $n \leq 5$. The inflection point between convex upward and downward profiles at the surface and center, respectively, moves toward the center from the surface for $n \geq 2$, and the centripetal motion terminates at $r={ }^{n} r_{\text {inf,nin, }}$ the final closest approaching distance at $t={ }^{n} t_{c, 0.99}$. It was found that ${ }^{n} r_{\text {inf,nin }} / a$ decreases with $n$ and is proportional to $n$ to the power of -0.354 . Because ${ }^{n} r_{\text {inf,nin }}$ reaches less than half of the radius for $n \geq 8$, ${ }^{n} r_{\text {inf,nin }} / a=n^{-0.354}$ could be used to numerically evaluate the degree of upward convexity in a profile. The ratio ${ }^{n} t_{f, 1.00} /{ }^{n} t_{c, 0.01}$ between the time ${ }^{n} t_{f, 1.00}$ when the surface gradient $|\partial T / \partial r|_{r=a}$ reaches $1.00 T_{s} / a$ to the time ${ }^{n} t_{c, 0.01}$ when the center temperature reaches $0.01 T_{\mathrm{s}}$ was found to evaluate the degree of upward convexity in the profile . The convex downward profile increases the center temperature first and then gradually increases the surface gradient toward a flat temperature profile; that is, ${ }^{n} t_{f, 1.00} /{ }^{n} t_{c, 0.01}$ is high. The strongly convex upward profile quickly achieves a flat surface gradient first; then, the center temperature gradually increases toward a flat 


\section{Nanomedicine \& Nanotechnology Open Access}

profile; that is, ${ }^{n} t_{\mathrm{f}, 1.00} /{ }^{n} t_{c, 0.01}$ is low. It was found that ${ }^{n} t_{\mathrm{f}, 1.00} /{ }^{n} t_{c, 0.01}$ decreases with $n$ and is proportional to $n$ to the power of -0.708 . Because ${ }^{n} t_{\mathrm{t}, 1.00} /{ }^{n} t_{\mathrm{c}, 0.01}$ is less than one for $n \geq 9,{ }^{n} t_{\mathrm{f}, 1.00} /{ }^{n} t_{c, 0.01}=4.44 n^{-0.708}$ could be used to numerically evaluate the degree of upward convexity in the profile. Although the surface gradient is attenuated exponentially with the time for any $n$, the time to achieve a flat center profile is biased to the final stage of the profile completion period for $n \geq 8$. This means that the heat transfer at the surface and center reflects planar and spherical geometry effects, respectively. However, the surface gradient and center temperature change exponentially with the time at the end of the final profile completion period, where the profile is almost flat, and the rates of change in the surface gradient and center temperature with the time are proportional to $n$ to the power of 1.51 and 1.57, respectively. This similarity in the rates of change at the surface and center means that the surface heat transfer, which is affected by the planar geometry, is also affected by the spherical geometry as one part of the sphere.

The attenuation of water rotation in spherical flasks was visualized by light-irradiated suspensions. Because the fluid rotation induced by shaking flasks is not actually the rotation of the outer shell, the final state just after shaking is not a fluid rotating with a uniform angular velocity, including the fluid surface. Thus, the duration of attenuation of the rotation was expected to be an intermediate value between the times ${ }^{5} t_{c, 0.01}$ and ${ }^{5} t_{c, 0.99}$ when the angular velocity at the center reaches 0.01 and 0.99 of the surface angular velocity, respectively. Although ${ }^{5} t_{c, 0.01}$ and ${ }^{5} t_{c, 0.99}$ are the growth periods of the thermal conduction of a five-dimensional sphere from the surface, the observed duration was distributed around the intermediate values between ${ }^{5} t_{c, 0.01}$ and ${ }^{5} t_{c, 0.99}$, and the proportionality to the square of the diameter was confirmed. The attenuation of water rotation in graduated cylinders was observed. When the water depth was higher than the diameter, the observed duration was distributed around the intermediate values between ${ }^{4} t_{c, 0.01}$ and ${ }^{4} t_{c, 0.99}$, and the proportionality to the square of the diameter was confirmed in a similar manner as the rotation in a spherical flask. When the water depth is lower than the diameter, the water rotation should be analyzed as rotation bounded by two coaxial disks. The observed duration was distributed around the intermediate values between ${ }^{1} t_{c, 0.01}$ and ${ }^{1} t_{c, 0.99}$, and the proportionality to the square of the height was confirmed in a similar manner as slab heating bounded by two parallel plates. It was found that the surface of the water in the graduated cylinder should be considered as an upper bottom and not a force-free boundary. Thus, the radius of the one-dimensional sphere corresponding to the water in the cylinder should be set as half of the depth in the calculations of ${ }^{1} t_{c, 0.01}$ and ${ }^{1} t_{c, 0.99}$ when the angular velocity at mid depth reaches 0.01 and 0.99 of the surface angular velocity, respectively. Although fluid rotation is induced by shaking and the spherical flask and graduated cylinder themselves could not be rotated, the observed durations of fluid rotation could be supported by the numerically determined durations of ${ }^{n} t_{c, 0.01}$ and ${ }^{n} t_{c, 0.99}$ for $n=1,4$, and 5. Therefore, it was concluded that the numerical simulation of the thermal conduction of a higher-dimensional sphere was useful for the analysis of fluid rotation.

\section{Acknowledgment}

The author is grateful to the late Dr. Ken'ichi Goto, Professor Emeritus of the Osaka University, for his insightful suggestions.

\section{References}

1. Osuga T, Sakamoto H, Takagi T (1996) Hydrodynamic analysis of electroosmotic flow in capillary. Journal of the Physical Society of Japan 65(6): 1854-1858.

2. Osuga T (1999) Comparison of growth of laminar flows in capillary: Poiseuille flow, electroosmotic flow, and electroosmotic circulation. Japanese Journal of Applied Physics 38(11): 6564-6567.

3. Melhem ER, Shakir H, Bakthavachalam S, MacDonald CB, Gira J, et al. (1998) Inner ear volumetric measurements using high-resolution $3 D T_{2}$-weighted fast Spin-echo MR imaging: Initial experience in healthy subjects. American Journal of Neuroradiological Research 19(10): 1819-1822.

4. ASTM F2119-07 (2013) Standard test method for evaluation of MR image artifacts from passive implants.

5. Wilkes JO (2010) Fluid mechanics for chemical engineering (Prentice Hall, Upper Saddle Riber).

6. Feynman RP, Leighton RB, Sands M (1963) The Feynman Lectures on Physics (Addison-Wesley, Massachusetts), Vol 1 and 2.

7. Lamb H (1945) Hydrodynamics (Dover, New York), p: 587. 


\section{Nanomedicine \& Nanotechnology Open Access}

8. Debye P (1929) Polar Molecules (Dover, New York), p: 84.

9. Osuga T, Tatsuoka H (2009) Magnetic field transfer of water molecules. Journal of Applied Physics 106(9): 094311.

10. Carslaw HS, Jaeger JC (1946) Conduction of heat in solids (Oxford University Press, New York).

11. Arpaci VS (1966) Conduction of heat transfer (Addison-Wesley, New York).

12. Osuga $\mathrm{T}$ (2000) Characteristic time required to achieve uniform temperature in thermal conduction of $n$-dimensional sphere heated from surface. Japan Journal of Applied Physics 39: 6111-6113.

13. von Neumann J, Richtmyer RD (1950) A method for the numerical calculation of hydrodynamic shocks. Journal of Applied Physics 21: 232-237.

14. Livne E, Glasner A (1985) A finite difference scheme for the heat conduction equation. Journal of Computational Physics 58: 59-66.
15. Courant R, Friedrichs KO, Lewy H (1928) Über die partiellen Differenzengleichungen der mathematischen Physik. Mathematische Annallen 100: 32-74.

16. Fromm JE (1968) A method for reducing dispersion in convective difference schemes. Journal of Computational Physics 3(2): 176-189.

17. Kubo R, Ichimura H, Usui T, Hashitsume N (1999) Statistical Mechanics (North- Holland, Amsterdam), p: 112.

18. Rayleigh L (1911) On the motion of solid bodies through viscous liquid. Philosophical Magazine 21(6): 697-711.

19. Batchelor GK (1967) An introduction to fluid dynamics (Cambridge University Press, Cambridge), Chap 4.

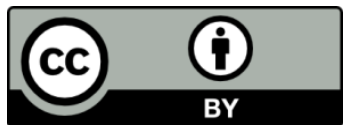

Osuga T. Thermal Conduction in Higher-Dimensional Sphere and Application to Fluid Rotation. Nanomed Nanotechnol 2017, 2(3): 